\title{
Appendix 1 - Chemistry: organic and trace metal data
}

The tables in this Appendix are the results of the chemical analyses of GEEP Workshop samples for the epibenthic sampling Sites 1 to 4 in Langesundfjord and the benthic community sampling Sites $A$ to $G$ in Frierfjord and Langesundfjord (Fig. 2 of Follum \& Moe 1988). Also given are the results for the 4 Exposure conditions $\mathrm{C}, \mathrm{L}, \mathrm{M}$ and $\mathrm{H}$ in the mesocosm experiment at Solbergstrand (Bakke et al. 1988). The following analyses were performed.

Tables 1, 3 to 10. Selected aromatic hydrocarbons (AHs) analysed by GC/MS in water, sediments, mussels and crabs from the mesocosm experiment, and AHs and PCBs in mussels and crabs from Field sites 1 to 4 , by Klungsøyr et al. (1988).

Table 2. Selected AHs analysed by GC/MS in sediments from Sites $A$ to $G$, by staff of the Norwegian Institute for Water Research (NIVA).

Table 11. Selected chlorinated hydrocarbons analysed in flounder liver from Field sites 1, 3 and 4, by Addison \& Edwards (1988).

Tables 12 to 18 . Trace metal concentrations analysed in sediments, mussels, crabs and flounder from field sites and mesocosm basins, by Abdullah \& Steffenak (1988).

The figures have been compiled (by K. R. Clarke, M. R. Carr and H. Archer) from the above tables and from additional chemical data in Livingstone (1988) and
Viarengo et al. (1988). Aromatic hydrocarbon data were totalled into 2- and 3-ring compounds (from naphthalene to $\mathrm{C}_{3}$-dibenzothiophenes in Table 1 , for example) and >3-ring compounds (from fluoranthene to perylene in Table 1 ).

As a result of variance-to-mean relationships in the original data, all values were log transformed before analysis. The pooled standard deviation from 1-way ANOVA was used to construct $95 \%$ confidence intervals; these, and the means, are displayed on the following figures on transformed axes (logs to the base e). Symbols without error bars are for single replicates. Symbol conventions for mesocosm samples are: (o) 'initial', (a) 'mid-term' and (+) 'final' samples; dosing of the $\mathrm{Cu}$ /diesel oil mixture commenced on 25 April. All sampling dates given are for 1986; 'August sampling' refers to field collections on 12 to 15 Aug and mesocosm collections on 18 to 21 Aug (11 Aug for $\mathrm{H}$ basin mussels)

In order to facilitate comparison, the same axis scaling has been adopted for equivalent field and mesocosm samples, and these figures are juxtaposed and have corresponding designations (e.g. c for field, $c^{\prime}$ for mesocosm). Unless stated otherwise, mesocosm figures are from the same source as the corresponding field plots. The papers cited in this Appendix are all from this MEPS SPECIAL.

Table 1. Water concentrations ( $\mathrm{ng}^{-1}$, detection limit $3 \mathrm{ng} \mathrm{l}^{-1}$ ) of selected PAHs from the mesocosm basins

\begin{tabular}{|c|c|c|c|c|c|c|c|c|c|c|}
\hline $\begin{array}{l}\text { Basin: } \\
\text { Sampling date }(1986) \text { : }\end{array}$ & 22 May & $\begin{array}{c}\mathrm{C} \\
19 \mathrm{Jun}\end{array}$ & $\stackrel{\mathrm{C}}{25 \mathrm{Jul}}$ & $22 \stackrel{L}{\text { May }}$ & $25 \mathrm{Jul}$ & $\begin{array}{c}\mathrm{M} \\
\text { 19 Jun }\end{array}$ & $\begin{array}{c}M \\
25 \mathrm{Jul}\end{array}$ & $\begin{array}{c}\mathrm{H} \\
22 \text { May }\end{array}$ & $\begin{array}{l}\mathrm{H} \\
19 \mathrm{Jun}\end{array}$ & $\begin{array}{c}\mathrm{H} \\
25 \mathrm{Jul}\end{array}$ \\
\hline Naphthaiene & 4 & 4 & 9 & 7 & 9 & 13 & 7 & 48 & 100 & 6 \\
\hline $\mathrm{C}_{1}$-Naphthalenes & 7 & 9 & 26 & 40 & 34 & 120 & 25 & 270 & 690 & 75 \\
\hline $\mathrm{C}_{2}$-Naphthalenes & 8 & 16 & 13 & 45 & 33 & 160 & 38 & 210 & 720 & 150 \\
\hline $\mathrm{C}_{3}$-Naphthalenes & 6 & 19 & 12 & 27 & 19 & 100 & 29 & 76 & 380 & 140 \\
\hline Anthracene & - & - & - & _- & - & - & - & - & - & - \\
\hline Phenantirene & 8 & 5 & _- & 3 & - & 15 & 3 & 11 & 45 & 8 \\
\hline$C_{1}$-Phenanthrenes & 10 & 15 & - & 5 & - & 35 & 8 & 23 & 130 & 32 \\
\hline $\mathrm{C}_{2}$-Phenanthrenes & 18 & 20 & - & 8 & - & 43 & 11 & 33 & 160 & 43 \\
\hline Dibenzothiophene & - & - & - & - & - & 6 & - & - & 21 & 6 \\
\hline$C_{1}$-Dibenzothiophenes & 7 & 3 & - & 3 & - & 14 & 4 & - & 49 & 15 \\
\hline $\mathrm{C}_{2}$-Dibenzothiophenes & 21 & 6 & - & 6 & - & 31 & 4 & 39 & 110 & 29 \\
\hline $\mathrm{C}_{3}$-Dibenzothiophenes & 18 & 4 & - & 3 & - & 11 & 3 & 29 & 48 & 16 \\
\hline Fluoranthene & 7 & - & - & - & - & - & - & - & 4 & - \\
\hline Pyrene & 28 & - & - & - & - & - & - & - & 8 & - \\
\hline Benz(a)anthracene & - & - & - & - & - & -- & - & - & - & - \\
\hline Chrysene & - & - & - & - & - & - & - & - & - & - \\
\hline Benz $(b+k)$ fluoranthene & - & _ & - & - & - & - & - & - & _- & - \\
\hline Benzie)pyrene & - & - & - & - & - & - & - & - & - & - \\
\hline Benzia)pyrene & - & - & - & - & - & - & - & - & - & - \\
\hline Perylene & - & - & - & - & - & - & - & - & - & - \\
\hline
\end{tabular}


Table 2. Sediment concentrations ( $\mathrm{ng} \mathrm{g}^{-1} \mathrm{dry}$ wt) of selected PAHs from the top $2 \mathrm{~cm}$ of Niemisto cores, taken at the macrofaunal sampling sites (A to $E, G$ ) on 22 Jan 1986 (2 replicate cores per site)

\begin{tabular}{|c|c|c|c|c|c|c|c|c|c|c|c|c|c|c|}
\hline Site: & A & A & B & B & $c$ & C & D & D & $\mathrm{E}$ & E & $\mathrm{F}$ & Fi & $G$ & $G$ \\
\hline Phenanthrene & 282 & 607 & 499 & 504 & 367 & 480 & 1322 & 577 & 312 & 260 & 2000 & 538 & 1333 & $41 \overline{8}$ \\
\hline Anthracene & 49 & 0 & 46 & 52 & 39 & 0 & 0 & 0 & 26 & 58 & 102 & 132 & 40 & 116 \\
\hline 2-Methylanthracene & 0 & 0 & 0 & 0 & 0 & 0 & 0 & 0 & 0 & 88 & 0 & 0 & 0 & 56 \\
\hline 1-Methylphenanthrene & 0 & 0 & 0 & 0 & 0 & 63 & 0 & 0 & 0 & 31 & 88 & 0 & 0 & 59 \\
\hline Fiuoranthene & 1829 & 891 & 319 & 815 & 227 & 599 & 1268 & 651 & 276 & 390 & 2406 & 1188 & 825 & 1113 \\
\hline Pyrene & 1536 & 702 & 280 & 807 & 192 & 367 & 1739 & 536 & 282 & 379 & 992 & 1138 & 539 & 1493 \\
\hline Benzolaifluorene & 0 & $\hat{0}$ & 0 & 122 & 0 & 0 & 0 & 0 & 52 & 173 & 228 & 274 & 0 & o \\
\hline Benzo(bifluorene & 0 & 0 & 63 & 171 & a & 55 & 0 & 0 & 42 & 183 & 220 & 270 & 0 & 0 \\
\hline 1-Methylpyrene & 0 & 0 & 0 & 0 & 0 & 66 & 0 & 0 & 47 & 0 & 177 & 73 & 0 & 0 \\
\hline Benzo(a)anthracene & 775 & 761 & 382 & 336 & 125 & 383 & 881 & 189 & 387 & 420 & 977 & 1538 & 612 & 1365 \\
\hline Triphenylerie/Chirysene & $95 ?$ & 871 & 658 & 529 & 209 & 746 & 1823 & 396 & 666 & 725 & 2596 & 3392 & 938 & 2011 \\
\hline Benzo $(b+j, k)$ fluoranthene & 323 & 1539 & 1948 & 1172 & 528 & 1107 & 2536 & 1082 & 1913 & 3161 & 4500 & 4494 & 1968 & 4525 \\
\hline Benzo(e)pyrene & 152 & 350 & 945 & 490 & 0 & 485 & 0 & 404 & 915 & 1096 & 0 & 1890 & 942 & 2115 \\
\hline Benzolapyrene & 150 & 300 & 1150 & 500 & 152 & 0 & 997 & 0 & 950 & 1000 & 1834 & 2456 & 2411 & 2300 \\
\hline Perylene & 0 & 0 & 0 & 0 & 0 & 0 & 0 & 0 & 0 & 242 & 0 & 0 & 0 & 515 \\
\hline O-Phenvlenepyrene & 155 & 349 & 588 & 354 & 388 & 506 & 0 & 341 & 756 & 745 & 1351 & 1395 & 768 & 845 \\
\hline Dibenz(a, h)anthracene & 0 & 136 & 192 & 62 & 128 & 0 & 0 & 146 & 227 & 248 & 0 & 328 & 106 & 389 \\
\hline Benzolghilperylene & 498 & 619 & 655 & 372 & 723 & 938 & 0 & 1027 & 718 & 725 & 1450 & 1144 & 427 & 1938 \\
\hline
\end{tabular}

Table 3. Sediment concentrations ( $\mathrm{ng} \mathrm{g}^{-1} \mathrm{dry} w \mathrm{w}$ ) of selected PAHs from the top $3 \mathrm{~cm}$ of box cores placed in the mesocosm basins on 17 Apr 1986 (5 boxes to each basin)

\begin{tabular}{|c|c|c|c|c|c|c|c|c|c|c|c|c|}
\hline Basin & $\mathrm{C}$ & $c$ & $c$ & $c$ & $c$ & $\mathrm{C}$ & $\mathrm{C}$ & C & $\mathrm{C}$ & $C$ & $\mathrm{C}$ & $\mathrm{C}$ \\
\hline Box number: & 1 & 1. & 2 & 2 & 3 & 3 & 4 & 4 & $\mathbb{1}$ & 2 & 3 & 4 \\
\hline Sampling date (1986): & $16 \mathrm{May}$ & $16 \mathrm{May}$ & 16 May & $16 \mathrm{May}$ & $16 \mathrm{May}$ & If May & 16 May & 16. May & 13 Jun & 13.Jun & $7 \mathrm{Jul}$ & $7 \mathrm{Jul}$ \\
\hline Naphthalene & 9 & 10 & 13 & 9 & 7 & 5 & 12 & 15 & 11 & 8 & 6 & 11 \\
\hline$C_{1}$-Naphthalenes & 12 & 11 & 15 & 12 & 9 & 6 & 12 & 16 & 12 & 9 & 7 & 13 \\
\hline $\mathrm{C}_{2} \cdot$ Naphthalenes & 22 & 25 & 42 & 30 & 10 & 11 & 22 & 28 & 29 & 16 & 13 & 20 \\
\hline $\mathrm{C}_{3}$-Napnthalenes & 18 & 17 & 18 & 22 & 12 & 10 & 17 & 23 & 17 & 13 & 11 & 19 \\
\hline Anthracene & 18 & 18 & 12 & 25 & 12 & 8 & 14 & 23 & 14 & 12 & 7 & 15 \\
\hline Phenanthrene & 6.3 & 51 & 38 & 76 & 46 & 30 & 47 & 72 & 49 & 39 & 30 & 50 \\
\hline$C_{1}$-Phenanthrenes & 51 & 43 & $5 !$ & 78 & 37 & 28 & 42 & 66 & 45 & 35 & 29 & 45 \\
\hline $\mathrm{C}_{2}$-Phenanthrenes & 45 & 44 & 25 & 35 & 33 & 28 & 48 & 60 & 44 & 38 & 30 & 51 \\
\hline Dibenzothiophene & 8 & 6 & 4 & 9 & 7 & 4 & 6 & 10 & 6 & 5 & 4 & 7 \\
\hline$C_{1}$-Dibenzothiophenes & 6 & 6 & 3 & 7 & 5 & 4 & 6 & 10 & 7 & 5 & 4 & 6 \\
\hline $\mathrm{C}_{2}$-Dibenzothiophenes & 17 & 18 & 15 & 15 & 13 & 11. & 20 & 25 & 20 & 15 & 13 & 20 \\
\hline $\mathrm{C}_{3}$-Dibenzothiophenes & 17 & 17 & 17 & 20 & 13 & 10 & 18 & 23 & 17 & 13 & 11 & 20 \\
\hline Fluoranthene & 120 & 110 & 90 & 150 & 89 & 71 & 1.10 & 150 & 120 & 97 & 73 & 110 \\
\hline Pyrene & 120 & 120 & 100 & 160 & 98 & 73 & 120 & 160 & 220 & 110 & 74 & 140 \\
\hline Benz(a)anthracene & 66 & 68 & 140 & 140 & 39 & 36 & 73 & 92 & 69 & 63 & 26 & 59 \\
\hline Chrysene & 63 & 51 & 120 & 120 & 36 & 33 & 54 & 60 & 53 & 48 & 28 & 47 \\
\hline Benz $(b+k)$ fluoranthene & 180 & 150 & 460 & 440 & 88 & 89 & 170 & 190 & 160 & 160 & 44 & 99 \\
\hline Benz(elpyrene & 120 & 64 & 230 & 300 & 57 & 50 & 88 & 1.20 & 84 & 90 & 45 & 77 \\
\hline Benz(a)pyrene & 64 & 38 & 580 & 170 & 26 & 25 & 50 & 63 & 44 & 45 & 21 & 48 \\
\hline Perylene & 41 & 23 & 96 & 92 & 19 & 16 & 28 & 35 & 30 & 28 & 14 & 25 \\
\hline
\end{tabular}

Table 3. (continued)

\begin{tabular}{|c|c|c|c|c|c|c|c|c|c|c|c|c|}
\hline Basin: & L & L & L & L & M & $\mathrm{M}$ & M & M & $H$ & $\mathrm{H}$ & $\mathrm{H}$ & $\mathrm{H}$ \\
\hline Bo\% number: & 1 & 2 & 3 & 4 & i & 2 & 3 & 4 & 1 & 2 & 3 & 4 \\
\hline Sampling date $\{1986\}$ : & 13 Jun & 13 Jun & $7 \mathrm{Jul}$ & 7 Jul & 13 Jun & 13 Juni & $7 \mathrm{Jul}$ & $7 \mathrm{Jul}$ & 13 Jun & 13 Jun & $7 \mathrm{Jul}$ & $7 \mathrm{Jul}$ \\
\hline Naphthalene & 12 & 15 & 8 & 12 & 13 & 7 & 16 & 14 & 11 & 10 & 14 & 10 \\
\hline$C_{1}$-Naphthalenes & 12 & 15 & 8 & 14 & 1.3 & 9 & 16 & 16 & 18 & 12 & 14 & 11. \\
\hline $\mathrm{C}_{2}$-Naphthalenes & 18 & 27 & 13 & 22 & 23 & 16 & 27 & 26 & 26 & 21 & 24 & 17 \\
\hline $\mathrm{C}_{3}$-Naphthalenes & 17 & 22 & 13 & 18 & 21 & 12 & 21 & 20 & 22 & 15 & 19 & 16 \\
\hline Anthracene & 16 & $\overline{2} i$ & 8 & 16 & 17 & 9 & 16 & 18 & 15 & 10 & 19 & 13 \\
\hline Phenanthrene & 52 & 60 & 35 & 56 & 58 & 31 & 59 & 56 & 47 & 37 & 59 & 45 \\
\hline C:-Phenanthrenes & 42 & 55 & 34 & 48 & 54 & 30 & 50 & 54 & 44 & 34 & 48 & 39 \\
\hline $\mathrm{C}_{2}$-Phenanthrenes & 46 & 56 & 33 & 48 & 57 & 30 & 51 & 55 & 48 & 35 & 49 & 41 \\
\hline Dibenzothiophene & 7 & 8 & 4 & 8 & 7 & 4 & 7 & 8 & 7 & 5 & 7 & 6 \\
\hline$C_{1}$-Dibenzothiophenes & 6 & 8 & 4 & 8 & 7 & 4 & 8 & 9 & 7 & 6 & 7 & 6 \\
\hline $\mathrm{C}_{3}$-Dibenzothophenes & 17 & 25 & 13 & 22 & 21 & 13 & $2 i$ & 23 & 18 & 16 & $18 \mathrm{i}$ & 17 \\
\hline$C_{1}$-Dibenzothiophenes & 14 & 23 & 11 & 20 & 18 & 12. & 20 & 22 & 16 & 17 & 17 & 15 \\
\hline Fluioranthene & 120 & 150 & 88 & 120 & 150 & 72 & 130 & 130 & 110 & 81 & 130 & 110 \\
\hline Pyiene & 140 & 150 & 81 & 130 & 160 & 80 & 140 & 150 & 150 & 90 & 150 & 120 \\
\hline Benzla)anthracene & 76 & 96 & 26 & 79 & 72 & 38 & 73 & 71 & 74 & 43 & 71 & 54 \\
\hline Chryseme & 53 & 67 & 24 & 68 & 57 & 34 & 63 & 63 & 54 & 40 & 61 & 43 \\
\hline Benz $(b+k)$ fluoranthene & 190 & 220 & 32 & 210 & 130 & 98 & 190 & 140 & 190 & 120 & 160 & 88 \\
\hline Benz(e)pyrene & 120 & 130 & 21 & 130 & 73 & 63. & 120 & 95 & 110 & 84 & 120 & 65 \\
\hline Benzia)pyrene & 57 & 64 & 11 & 75 & 42 & 27 & 68 & 55 & 53 & $4 !$ & 74 & 40 \\
\hline Perylene & 29 & 40 & 7 & 53 & 23 & 31 & 41 & 31 & 28 & 26 & 32 & 19 \\
\hline
\end{tabular}


Table 4. Mytilus edulis. Whole tissue concentrations (ng $\mathrm{g}^{-1}$ dry wt) of selected PAHs in mussels from Field sites 1 to 4 (pool of 10 ind sample ${ }^{-1}$ )

\begin{tabular}{|c|c|c|c|c|c|c|c|c|}
\hline $\begin{array}{l}\text { Site: } \\
\text { Sampling clate (1986): }\end{array}$ & $\begin{array}{c}1 \\
\text { if Aug }\end{array}$ & 11 Aug & 13 Aug & 13 Aug & $\begin{array}{l}3 \\
12 \mathrm{Augg}\end{array}$ & $12 \stackrel{3}{\mathrm{~A} u \mathrm{~g}}$ & $\begin{array}{c}4 \\
14 \text { Ang }\end{array}$ & 14 Ang \\
\hline Naphthalene & 85 & $5 !$ & 15 & 17 & 22 & 22 & 17 & 24 \\
\hline $\mathrm{C}_{1}$-Naphthalenes & 41 & 36 & 26 & 31 & 39 & 39 & 31 & 39 \\
\hline C -Naphthalenes & 64 & 62 & 57 & 63 & 79 & 63 & 70 & 86 \\
\hline$C_{3}$ Naphthalenes & 78 & 87 & 110 & 100 & 130 & 110 & 150 & 200 \\
\hline Anthracene & 17 & 15 & 26 & 22 & 71 & 49 & 62 & 57 \\
\hline Phenanthrene & 170 & 160 & 310 & 320 & 660 & 600 & 490 & 620 \\
\hline C:-Phenanthrenes & 150 & 130 & 300 & 300 & 460 & 420 & 460 & $6 \mathrm{~L} 0$ \\
\hline $\mathrm{C}_{2}$-Phenanthrenes & 160 & 170 & 410 & 420 & 610 & 550 & 820 & 1100 \\
\hline Dibenzothiophene & 18 & 17 & 33 & 36 & 62 & 59 & 50 & 64 \\
\hline C-Dibenzothiophenes & 28 & 24 & 48 & 47 & 72 & 70 & 76 & 100 \\
\hline $\mathrm{C}_{2}$-Dibenzothiophenes & 93 & 81 & 200 & 190 & 300 & 290 & 410 & 640 \\
\hline$C_{2}$-Dibenzothiophenes & 64 & 60 & 150 & 140 & 240 & 230 & 420 & 650 \\
\hline Fluoranthene & 680 & 630 & 2200 & 2200 & 3900 & 3500 & 3300 & 4300 \\
\hline Pyrene & 140 & 140 & 390 & 320 & 1200 & 910 & 1100 & 1300 \\
\hline Benzla)unthracene & 110 & 98 & 320 & 230 & 670 & 560 & 1500 & 1200 \\
\hline Chrysene & 250 & 210 & 880 & 860 & 1500 & 1600 & 2600 & 2700 \\
\hline Benz $(b+k)$ fluoranthene & 110 & 120 & 330 & 300 & 1100 & 960 & 1600 & 1900 \\
\hline Benzlejpyrene & 46 & 44 & 160 & 110 & 750 & 660 & 590 & 1000 \\
\hline Eenz(a)pyrene & 14 & 16 & 29 & 32 & 150 & 130 & 230 & 240 \\
\hline Perylene & 4 & 4 & 8 & 7 & 45 & 40 & 60 & 68 \\
\hline
\end{tabular}

Table 5. Carcinus maenas. Whole tissue concentrations ( $\mathrm{ng} \mathrm{g}^{-1}$ dry wt, detection limit $1 \mathrm{ng} \mathrm{g}^{-1}$ ) of selected PAHs in crabs from Field sites 1 to 4 (pool of 4 ind. sample ${ }^{-1}$ )

\begin{tabular}{|c|c|c|c|c|c|c|c|c|}
\hline $\begin{array}{l}\text { Site: } \\
\text { Sampling date (1986): }\end{array}$ & $11 \stackrel{1}{\text { Aug }}$ & $\begin{array}{c}1 \\
n \text { Aug }\end{array}$ & $\begin{array}{l}2 \\
13 \text { Aug }\end{array}$ & $\begin{array}{c}2 \\
13 \text { Aug }\end{array}$ & $12 \stackrel{3}{\text { Aug }}$ & $12 \stackrel{3}{\text { Aug }}$ & $14 \stackrel{4}{\text { Aug }}$ & $\begin{array}{l}4 \\
14 \text { Aug }\end{array}$ \\
\hline Naphthalene & 12 & 10 & 10 & 14 & 29 & 24 & 10 & 23 \\
\hline$C_{1}-$ Naphthalenes & 18 & 17 & 14 & 18 & 32 & 22 & 14 & 27 \\
\hline$C_{2}$ - Naphthalenes & 8 & 14 & 12 & 13 & 32 & 23 & 11 & 22 \\
\hline$C_{3}-$ Naphthalenes & - & - & 7 & 7 & 10 & 12 & 7 & 12 \\
\hline Anthracene & - & - & - & - & - & - & - & - \\
\hline Phenanthrene & 12 & 11 & 15 & 11 & 38 & 20 & 29 & 32 \\
\hline$C_{1}$-Phenanthrenes & 6 & 5 & 6 & 7 & 12 & 10 & 10 & 13 \\
\hline$C_{2}$-Phenanthrenes & - & - & 6 & - & - & - & 11 & 18 \\
\hline Dibenzothiophene & - & - & 3 & 2 & 7 & - & - & 4 \\
\hline$C_{1}$-Dibenzathiophenes & - & - & - & - & - & - & - & - \\
\hline $\mathrm{C}_{2}$-Dibenzothiopheres & - & - & - & - & - & - & - & - \\
\hline$C_{1}$-Dibenzothophenes & - & - & - & - & - & - & - & - \\
\hline Fluoranthene & 14 & 11 & 18 & 14 & 26 & 15 & 46 & 55 \\
\hline Pyrenc & 5 & 5 & 7 & 4 & 10 & 6 & 15 & 21 \\
\hline Benz(a)anthracene & - & - & 6 & 7 & - & 9 & 13 & 11 \\
\hline Chrysene & 5 & - & 9 & 8 & 8 & 8 & 25 & 30 \\
\hline Benz $(b+k)$ fluorantiene & - & - & 6 & - & - & - & 12 & 17 \\
\hline Benzielpyrene & - & - & 3 & - & - & - & - & - \\
\hline Benz:aipyrene & - & - & - & - & - & - & - & \\
\hline Perylene & - & - & - & - & - & - & - & - \\
\hline
\end{tabular}

Table 6. Mytilus edulis. Whole tissue concentrations ( $\mathrm{ng} \mathrm{g}^{-1} \mathrm{dry} w \mathrm{w}$, detection limit $1 \mathrm{ng} \mathrm{g}^{-1}$ ) of selected PAHs in mussels from cages originally stocked in mesocosm basins (25 Apr 1986, 2 cages basin ${ }^{-1}$, pool of 10 ind. sample ${ }^{-1}$ )

\begin{tabular}{|c|c|c|c|c|c|c|c|c|c|c|c|c|c|c|c|c|}
\hline Basin: & C & C & C & C & $c$ & c & 1. & $L$ & L & $\mathrm{L}$ & $M$ & M & M & $M$ & $\mathrm{H}$ & $\mathrm{H}$ \\
\hline Cage number: & 1 & 2 & 1 & 2 & 1 & 2 & 1 & 2 & 1 & 2 & 1 & 2 & 1 & 2 & 1 & 2 \\
\hline Sampling date (1986): & 16 May & 16 May & $13 \mathrm{Jish}$ & $13 \mathrm{Jun}$ & 4 Aug & 4 Aug & $13 \mathrm{Jun}$ & $13 \mathrm{Jun}$ & 4 Aug & 4 Aug & 13 Jun & 13 Jun & $4 \mathrm{Aug}$ & 4 Aug & $13 \mathrm{Jun}$ & $13 \mathrm{Jun}$ \\
\hline Naphthalene & 4 & 3 & 6 & 5 & 12 & 13 & 7 & 6 & 20 & 21 & 10 & 11 & 9 & 16 & 260 & 250 \\
\hline $\mathrm{C}_{\mathrm{i}}$-Naphthalenes & 23 & 28 & 26 & 28 & 78 & 88 & 43 & 53 & 150 & 140 & 150 & 270 & 230 & 250 & 4900 & 4400 \\
\hline $\mathrm{C}_{2}$-Naphthalenes & 51 & 67 & 53 & 51 & 140 & 170 & 220 & 250 & 470 & 440 & 1100 & 1600 & 1300 & 1300 & 9500 & 7700 \\
\hline $\mathrm{C}_{3}$-Naphthalenes & 73 & 100 & 66 & 76 & 140 & 180 & 510 & 710 & 940 & 870 & 2800 & 4000 & 3600 & 3200 & 7000 & 5200 \\
\hline Anthracene & 2 & 3 & - & 1 & - & - & 4 & 4 & 3 & 3 & 10 & 25 & 11 & 13 & 26 & 19 \\
\hline Phenanthrene & 13 & 17 & 10 & 12 & 10 & 13 & 38 & 37 & 38 & 33 & 140 & 210 & 130 & 150 & 550 & 450 \\
\hline$C_{1}$-Phenanthrenes & 64 & 79 & 48 & 60 & 59 & 73 & 500 & 550 & 470 & 450 & 1700 & 2400 & 2200 & 2100 & 1900 & 1600 \\
\hline $\mathrm{C}_{2}$-Phenanthrenes & 170 & 210 & 130 & 170 & 150 & 170 & 1600 & 1600 & 1600 & 1500 & 4000 & 4600 & 5700 & 6300 & 2000 & 1700 \\
\hline Dibenzothiophene & 2 & 2 & 2 & 1 & 1 & 2 & 30 & 25 & 12 & 12 & 75 & 100 & 98 & 96 & 240 & 200 \\
\hline$C_{1}$-Dibenzothiophenes & 24 & 27 & 20 & 23 & 23 & 28 & 190 & 220 & 140 & 140 & 740 & 1000 & 800 & 890 & 770 & 590 \\
\hline$C_{2}$-Dibenzothiophenes & 160 & 190 & 110 & 160 & 140 & 160 & 1300 & 1300 & 1300 & 1300 & 3000 & 3500 & 4500 & 4600 & 1600 & 1300 \\
\hline $\mathrm{C}_{3}$-Dibenzothiophenes & 130 & 150 & 120 & 140 & 130 & 160 & 1100 & 900 & 1100 & 1000 & 2000 & 2200 & 3700 & 3800 & 590 & 510 \\
\hline Fluoranthene & 84 & 110 & 55 & 78 & 37 & 36 & 77 & 73 & 51 & 37 & 95 & 130 & 67 & 79 & 190 & 150 \\
\hline Pyrene: & 33 & 42 & 27 & 36 & 25 & 23 & 48 & 47 & 36 & 36 & 71 & 89 & 63 & 73 & 79 & 67 \\
\hline Benz(a)anthracene & 5 & 5 & 6 & 3 & 3 & 3 & 5 & 6 & 2 & 3 & 7 & 7 & 7 & 7 & 3 & 7 \\
\hline Chrysene & 41 & 42 & 42 & 38 & 24 & 24 & 37 & 44 & 28 & 25 & 47 & 57 & 58 & 74 & 64 & 82 \\
\hline Benz $(b+k) f$ luoranthene & 8 & 11 & 12 & 8 & 6 & 7 & 8 & 11 & 7 & 4 & 6 & 9 & 5 & 17 & 10 & 20 \\
\hline Benzleipyrene & 14 & 22 & 19 & 21 & 15 & 15 & 11 & 17 & 17 & 10 & 9 & 17 & 14 & 27 & 39 & 37 \\
\hline Benz(a)pyrene & - & - & - & - & - & - & - & 1 & - & - & - & - & - & - & - & - \\
\hline Perylene & - & - & - & - & - & - & - & - & - & - & - & - & - & - & - & - \\
\hline
\end{tabular}


Table 7. Mytilus edulis. Whole tissue concentrations ( $\mathrm{ng} \mathrm{g}^{-1} \mathrm{dry}$ wt, detection limit $1 \mathrm{ng} \mathrm{g}^{-1}$ ) of selected PAHs in mussels from the later restocking of mesocosm basins ( $18 \mathrm{Jul}$ 1986, a further 2 cages basin ${ }^{-1}$, pool of $10 \mathrm{ind}$. sample ${ }^{-1}$ ). $\mathrm{T}_{0}$ readings are from this population, prior to stocking in the basins

\begin{tabular}{|c|c|c|c|c|c|c|c|c|c|c|}
\hline Basin: & $T_{0}$ & $T_{0}$ & C & C & $\mathrm{L}$. & ! & $M$ & $M$ & $\mathrm{H}$ & $\mathrm{H}$ \\
\hline Cage number: & - & - & 3 & 4 & 3 & 4 & 3 & 4 & 3 & 4 \\
\hline Sampling date (1986): & $7 \mathrm{Jul}$ & $7 \mathrm{Jul}$ & 4 Aug & 4 Aug & 4 Aug & 4 Aug & 4 Aug & 4 Aug & 4 Aug & 4 Aug \\
\hline Naphthalene & 5 & 2 & 12 & 9 & 16 & 21 & 13 & 13 & 32 & 22 \\
\hline $\mathrm{C}_{\mathrm{t}}$-Naphthalenes & 2.5 & 8 & 75 & 61 & 130 & 180 & 200 & 200 & 480 & 680 \\
\hline $\mathrm{C}_{2}$-Naphthalenes & 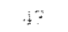 & 35 & 140 & 120 & 410 & 290 & 1000 & 1200 & 2400 & 2600 \\
\hline $\mathrm{C}_{3}$-Naphthalenes & 40 & 37 & 130 & 120 & 670 & 730 & 2500 & 2700 & 2500 & 2700 \\
\hline Anthracene & - & 1 & - & - & 2 & 2 & 9 & 8 & 8 & 10 \\
\hline Phenanthrene & 17 & 18 & 9 & 9 & 32 & 36 & 110 & 110 & 120 & 180 \\
\hline$C_{1}$-Phenanthrenes & 23 & 22 & 42 & 41 & 290 & 290 & 1200 & 1200 & 520 & 820 \\
\hline $\mathrm{C}_{2}$-Phenanthrenes & 35 & 31 & 79 & 74 & 690 & 580 & 3100 & 2700 & 500 & 800 \\
\hline Dibenzothiophene & 2 & 2 & 1 & 1 & 10 & 14 & 40 & 42 & 51 & 87 \\
\hline$C_{1}$-Dibenzothiophenes & $?$ & 8 & 15 & $\$ 1$ & 120 & 120 & 420 & 540 & 230 & 340 \\
\hline $\mathrm{C}_{2}$-Dibenzothiophenes & 25 & 19 & 62 & 59 & 560 & 470 & 2300 & 2100 & 320 & 570 \\
\hline $\mathrm{C}_{3}$-Dibenzothiophenes & 22 & 17 & 38 & 40 & 340 & 270 & 1300 & 1200 & 110 & 170 \\
\hline Fluoranthene & 8 & 8 & 17 & 14 & 19 & 24 & 34 & 28 & 17 & 2.8 \\
\hline Pyrene & 7 & 7 & 14 & 13 & 20 & 23 & 51 & 45 & 16 & 26 \\
\hline Benziajanthracene & : & 2 & 1 & 2 & 2 & 1 & 4 & 4 & 1 & 1 \\
\hline Chrysere & $\therefore$ & 4 & 8 & 8 & 11 & 9 & 17 & 17 & 4 & 5 \\
\hline Benz $(b+k)$ fluoranthene & 2 & 3 & 2 & 1 & 2 & 2 & 2 & - & 1 & - \\
\hline Benz(e)pyrene & 3 & 4 & 4 & 2 & 6 & 5 & 3 & - & 3 & - \\
\hline Benzlalpyrene & - & - & - & - & - & - & - & - & - & - \\
\hline Perylene & - & - & - & - & - & - & - & - & - & - \\
\hline
\end{tabular}

Table 8. Carcinus maenas. Whole tissue concentrations (ng $\mathrm{g}^{-1}$ dry wt, detection limit $1 \mathrm{ng} \mathrm{g}^{1}$ ) of selected PAHs in crabs from mesocosm basins (placed in basins on 12 May 1986; pool of 4 ind. sample ${ }^{-1}$ )

\begin{tabular}{|c|c|c|c|c|c|c|c|c|}
\hline $\begin{array}{l}\text { Basin: } \\
\text { Sampling date (1986): }\end{array}$ & $\begin{array}{c}\text { C } \\
4 \text { Aug }\end{array}$ & $\begin{array}{c}\text { C } \\
4 \text { Aug }\end{array}$ & $\underset{4 \mathrm{Aing}}{\mathrm{L}}$ & $\begin{array}{c}\mathrm{L} \\
4 \text { Aug }\end{array}$ & $\begin{array}{c}\text { M } \\
4 \text { Aug }\end{array}$ & $\begin{array}{c}\mathrm{M} \\
4 \mathrm{Aug}\end{array}$ & $\begin{array}{c}\mathrm{H} \\
4 \mathrm{Aug}\end{array}$ & $\begin{array}{c}\mathrm{H} \\
4 \mathrm{Aug}\end{array}$ \\
\hline Naphthalene & 10 & 11 & 26 & 12 & 70 & 36 & 37 & 37 \\
\hline$C_{1}$-Naphthalenes & 64 & 35 & 130 & 91 & 240 & 280 & 770 & 910 \\
\hline $\mathrm{C}_{2}-\mathrm{Naphthalenes}$ & 34 & 18 & 89 & 71 & 83 & 190 & 1300 & 1300 \\
\hline $\mathrm{C}_{3}=$ Naphthalenes & 6 & 9 & 30 & 17 & 34 & 44 & 510 & 340 \\
\hline Anthracene & - & - & $=$ & - & - & - & $\hat{\imath}$ & 2 \\
\hline Phenanthrene & 2 & 3 & 8 & 2 & 9 & 3 & 34 & 29 \\
\hline$C_{1}$-Phenanthrenes & 2 & 3 & 8 & 5 & 12 & 12 & 65 & 45 \\
\hline $\mathrm{C}_{2}$-Phenanthrenes & - & - & 9 & 10 & 11 & 24 & 58 & 44 \\
\hline Dibenzothiophene & - & - & 1 & 1 & 2 & 3 & 34 & 28 \\
\hline$C_{1}$-Dibenzothiophenes & - & - & 4 & 2 & 5 & 7 & 40 & 28 \\
\hline$C_{2}$-Dibenzothiophenes & - & - & 9 & 6 & 9 & 17 & 42 & 36 \\
\hline $\mathrm{C}_{3}$-Dibenzothiophenes & - & - & 6 & 4 & 7 & 15 & 17 & 19 \\
\hline Fluoranthene & - & - & j & - & 1 & - & 1 & 1 \\
\hline Fyrene & - & - & - & - & 1 & - & 2 & 1 \\
\hline Berz(a) anthracene & - & - & - & - & - & - & - & - \\
\hline Chrysene & - & - & - & - & - & - & - & - \\
\hline Benz $(b+k)$ fluoranthene & - & - & - & - & - & - & - & - \\
\hline Benz(e)pyrene & - & - & - & - & - & - & - & - \\
\hline Benz(a)pyrene & - & - & - & - & - & - & - & - \\
\hline Perylene & - & - & - & - & - & - & - & - \\
\hline
\end{tabular}

Table 9. Mytilus edulis. Whole tissue concentrations $\left(\mathrm{ng}^{-1}\right.$ dry wt, detection limit $0.1 \mathrm{ng} \mathrm{g}^{-1}$ ) of selected PCB congeners, and total PCBs, in mussels from Field sites 1 to 4 (pool of 10 ind sample ${ }^{-1}$ ). IUPAC 53 used as internal standard

\begin{tabular}{|c|c|c|c|c|c|c|c|c|}
\hline Site: & 1 & 1 & 2 & 2 & 3 & 3 & 4 & 4 \\
\hline $\begin{array}{l}\text { Date } \\
(1986):\end{array}$ & 11 Aug & I1 Aug & $13 \mathrm{Aug}$ & $13 \mathrm{Aug}$ & 12 Aug & $12 \mathrm{Aug}$ & 14 Aug & $14 \mathrm{Aug}$ \\
\hline \multicolumn{9}{|l|}{ IUPAC } \\
\hline 52 & 9.3 & 9.0 & 46.0 & 42.0 & 57.0 & 69.0 & 63.0 & 69.0 \\
\hline 44 & 28 & 26 & 3.2 & 3.5 & 30 & 30 & 43 & 5.8 \\
\hline 95 & 4.3 & 3.4 & 53 & 6.3 & 6.6 & 6.9 & 32 & 120 \\
\hline 101 & 5.1 & 46 & 8.1 & 9.7 & 10.0 & 11.0 & 13.0 & 160 \\
\hline 110 & 4.1 & 3.2 & 8.1 & 4.5 & 8.1 & 89 & 11.0 & 15.0 \\
\hline 118 & 4.8 & 3.8 & 7.7 & 9.5 & 92 & 11.0 & 14.0 & 18.0 \\
\hline 153 & 7.8 & 7.0 & 130 & 150 & 15.0 & 18.0 & 17.0 & 19.0 \\
\hline $138:$ & 6.9 & 5.9 & 11.0 & 13.0 & 13.0 & 15.0 & 16.0 & 19.0 \\
\hline 128 & 1.1 & 1.1 & 1.7 & 2.6 & 2.8 & 3.0 & 4.2 & 3.7 \\
\hline 180 & 1.2 & 0.8 & 2.3 & 4.1 & 4.3 & 5.1 & 4.9 & 5.8 \\
\hline 170 & 0.4 & - & 13 & 2.0 & 13 & 1.6 & 2.1 & 21 \\
\hline 194 & - & - & 0.9 & 14 & 0.8 & 1.0 & 1.2 & 16 \\
\hline \multicolumn{9}{|l|}{ Total } \\
\hline PCBs & 83 & 70 & 170 & 190 & 210 & 240 & 250 & 300 \\
\hline
\end{tabular}

Table 10. Carcinus maenas. Whole tissue concentrations (ng $\mathrm{g}^{-1}$ dry wt) of selected PCB congeners, and total PCBs, in crabs from Field sites 1 to 4 (pool of 4 ind. sample ${ }^{-1}$ )

\begin{tabular}{|c|c|c|c|c|c|c|c|c|}
\hline $\begin{array}{l}\text { Site: } \\
\text { Date } \\
\text { (1986): }\end{array}$ & 11 A Aug & $\begin{array}{c}1 \\
\text { if Àug }\end{array}$ & $13 \stackrel{2}{\text { Aug }}$ & $13 \stackrel{2}{\text { Aug }}$ & $\begin{array}{c}3 \\
12 \mathrm{Aug}\end{array}$ & $\stackrel{3}{\mathrm{Aug}}$ & $14 \stackrel{4}{\text { Aug }}$ & $14 \stackrel{4}{\text { Aug }}$ \\
\hline \multicolumn{9}{|l|}{ IUPAC } \\
\hline 52 & 7.7 & 6.0 & 11.0 & 120 & 11.0 & 11.0 & 180 & 24.0 \\
\hline 44 & 1.6 & 1.0 & 2.1 & 2.5 & 2.4 & 2.0 & 2.3 & 4.3 \\
\hline 95 & 5.8 & 4.7 & 16.0 & 130 & 11.0 & 10.0 & 17.0 & 35.0 \\
\hline $10:$ & 11.0 & 10.0 & 22.0 & 19.0 & 16.0 & 17.0 & 23.0 & 26.0 \\
\hline 110 & 3.8 & 33 & 7.9 & 9.7 & 7.7 & 7.7 & 11.0 & 15.0 \\
\hline 118 & 11.0 & 11.0 & 28.0 & 22.0 & 21.0 & 20.0 & 34.0 & 51.0 \\
\hline 153 & 20.0 & 210 & 37.0 & 31.0 & 33.0 & 39.0 & 40.0 & 43.0 \\
\hline 138 & 17.0 & 170 & 32.0 & 28.0 & 270 & 31.0 & 36.0 & 43.0 \\
\hline 128 & 2.5 & 22 & 44 & 3.9 & 3.2 & 3.5 & 5.6 & 5.6 \\
\hline 180 & 4.2 & 4.4 & 100 & 8.6 & 16.0 & 160 & 16.0 & 21.0 \\
\hline 170 & 1.7 & 1.6 & 3.9 & 4.7 & 9.2 & 93 & 7.4 & 11.0 \\
\hline 194 & 1.2 & 1.2 & 2.3 & 2.5 & 5.2 & 4.8 & 3.4 & 5.6 \\
\hline \multicolumn{9}{|l|}{ Total } \\
\hline PCBs & 160 & 160 & 340 & 300 & 330 & 350 & 410 & 550 \\
\hline
\end{tabular}


Table 11. Platichthys flesus. Liver concentrations ( $\mu \mathrm{g} \mathrm{g}^{-1}$ wet $w \mathrm{t}$ ) of selected PCB congeners (and the total of these congeners), hexachlorobenzene and octachlorostyrene in fish from 3 field sites (mean \pm SD, from $n=5,10,11$ fish from Sites, $1,3,4$ respectively)

\begin{tabular}{|c|c|c|c|c|}
\hline \multicolumn{2}{|l|}{$\begin{array}{l}\text { Ste: } \\
\text { Sampling date (1986): }\end{array}$} & $\begin{array}{c}1 \\
11-12 \text { Aug }\end{array}$ & $\begin{array}{c}3 \\
12-13 \text { Aug }\end{array}$ & $\begin{array}{c}4 \\
14-15\end{array}$ \\
\hline \multirow[t]{28}{*}{ IUPAC number } & 31 & $0.000 \pm 0.000$ & $1.535 \pm 0.794$ & $1.851 \pm 1.974$ \\
\hline & 87 & $0.025 \pm 0.050$ & $0.000 \pm 0.000$ & $0.000 \pm 0.000$ \\
\hline & 60 & $0.000=0.000$ & $0.055 \pm 0.094$ & $0.002 \pm 0.008$ \\
\hline & 101 & $0.128 \pm 0.046$ & $0.060 \pm 0.063$ & $0.029 \pm 0.020$ \\
\hline & 154 & $0.000 \pm 0.000$ & $0.000: \pm 0.000$ & $0.003 \pm 0.008$ \\
\hline & 151 & $0.010 \pm 0.020$ & $0.023:=0.029$ & $0.005 \pm 0.009$ \\
\hline & 118 & $0.000 \pm 0.000$ & $0.040 \pm 0.063$ & $0.029 \pm 0.026$ \\
\hline & 153 & $0.066 \pm 0.022$ & $0.077 \pm 0.040$ & $0.084 \pm 0.081$ \\
\hline & 141 & $0.011 \pm 0014$ & $0.024 \pm 0.028$ & $0.005 \pm 0.009$ \\
\hline & 137 & $0.000 \pm 0.000$ & $0016 \pm 0033$ & $0008 \pm 0024$ \\
\hline & 138 & $0.032 \pm 0.020$ & $0.053=0.057$ & $0.081 \pm 0.053$ \\
\hline & 187 & $0.000 \pm 0.000$ & $0.034: \pm 0.014$ & $0.022 \pm 0.018$ \\
\hline & 159 & $0.007 \pm 0.009$ & $0.043 \pm 0.018$ & $0.046 \pm 0.046$ \\
\hline & 182 & $0.000 \pm 0.000$ & $0.035 \pm 0.015$ & $0.036 \pm 0.039$ \\
\hline & 183 & $0.000 \pm 0.000$ & $0.017 \pm 0.021$ & $0.029 \pm 0.041$ \\
\hline & 156 & $0.000 \pm 0.000$ & $0.021 \pm 0.022$ & $0.018=0.029$ \\
\hline & 202 & $0.000 \pm 0.000$ & $0.015 \pm 0.015$ & $0.013 \pm 0.022$ \\
\hline & 200 & $0.000: \pm 0.000$ & $0.052 \pm 0.028$ & $0.050 \pm 0.041$ \\
\hline & 180 & $0.008 \pm 0.011$ & $0.006 \pm 0.013$ & $0.016 \pm 0016$ \\
\hline & 191 & $0.000 \pm 0.000$ & $0.007 \pm 0.014$ & $0.001=0.003$ \\
\hline & 170 & $0.033 \pm 0.041$ & $0.024 \pm 0.036$ & $0.007 \pm 0.016$ \\
\hline & 201 & $0.005 \pm 0.010$ & $0.014 \pm 0.020$ & $0.022 \pm 0.033$ \\
\hline & 203 & $0005 \pm 0006$ & $0.039 \pm 0.025$ & $0.078 \pm 0.073$ \\
\hline & 196 & $0.004 \pm 0.007$ & $0.051 \pm 0.032$ & $0.047 \pm 0.037$ \\
\hline & 208 & $0.003 \pm 0.006$ & $0.054 \pm 0.020$ & $0.047 \pm 0.038$ \\
\hline & 207 & $0.030 \pm 0.016$ & $0.138=0.066$ & $0.124 \pm 0.111$ \\
\hline & 206 & $0.011 \pm 0.009$ & $0.081 \pm 0.063$ & $0.072 \pm 0.076$ \\
\hline & 209 & $0.133 \pm 0.011$ & $0.536 \pm 0.271$ & $0.827 \pm 1.417$ \\
\hline Total & & $0.511 \quad 0.132$ & $3.049 \quad 1.502$ & $3.551 \quad 3.166$ \\
\hline Hexachlorobenze & & $0236 \quad 0.243$ & $1.800 \quad 1.099$ & 1.372 \\
\hline Oclachiorostyrene & & $0063 \quad 0.018$ & $0.944 \quad 1.231$ & $0.710 \quad 1.023$ \\
\hline
\end{tabular}

Table 12. Trace metal concentrations $\left(\mu \mathrm{g} \mathrm{g} \mathrm{g}^{-1}\right.$ dry wt, Fe as $\%$ dry wt) in the top $2 \mathrm{~cm}$ of sediments (size fraction $<63 \mu \mathrm{m}$ ) from 3 replicate cores at the benthic macrofaunal sampling sites (A to G) in Frierfjord/Langesundfjord

\begin{tabular}{|c|c|c|c|c|c|c|c|c|}
\hline Site & $\mathrm{Cu}$ & $\mathrm{Zn}$ & $\mathrm{Pb}$ & $N_{i}$ & $\mathrm{Cr}$ & $\mathrm{Cd}$ & $\mathrm{Mn}$ & $\mathrm{Fe}$ \\
\hline \multirow[t]{3}{*}{ A } & 28 & 141 & 73 & 33 & 40 & 0.8 & 454 & 35 \\
\hline & 26 & 139 & 71 & 30 & 40 & $(0.6)$ & 653 & 3.3 \\
\hline & 27 & 147 & 67 & 29 & 35 & $(0.6)$ & 503 & 3.1 \\
\hline \multirow[t]{3}{*}{ B } & 48 & 238 & 134 & 33 & 50 & $(0.6)$ & 1050 & 3.5 \\
\hline & 47 & 228 & 130 & 32 & 50 & 11 & 2880 & 3.5 \\
\hline & 64 & 297 & 167 & 32 & 40 & 11 & 664 & 3.1 \\
\hline \multirow[t]{3}{*}{ C } & 44 & 228 & 135 & 35 & 51 & 0.8 & 1500 & 4.1 \\
\hline & 42 & 216 & 126 & 35 & 60 & 0.8 & 3570 & 42 \\
\hline & 42 & 208 & 117 & 33 & 45 & 1.1 & 5880 & 4.0 \\
\hline \multirow[t]{3}{*}{ D } & 48 & 241 & 142 & 37 & 56 & 0.9 & 1720 & 4.3 \\
\hline & 39 & 205 & 114 & 33 & 50 & 0.8 & 8480 & 44 \\
\hline & 44 & 238 & 141 & 35 & 34 & 1.1 & 5440 & 4.1 \\
\hline \multirow[t]{3}{*}{$E$} & 38 & 199 & 160 & 22 & 40 & 0.8 & 484 & 2.2 \\
\hline & 40 & 241 & 156 & 25 & 40 & 1.1 & 925 & 2.1 \\
\hline & 107 & 275 & 184 & 28 & 45 & 1.1 & 1400 & 25 \\
\hline \multirow[t]{3}{*}{ F } & 48 & 328 & 118 & 32 & 35 & 3.6 & 10380 & 31 \\
\hline & 44 & 296 & 110 & 30 & 35 & 3.1 & 5880 & 30 \\
\hline & 47 & 320 & 118 & 32 & 35 & 34 & 7430 & 3.0 \\
\hline \multirow[t]{3}{*}{$G$} & 67 & 349 & 212 & 35 & 61 & 22 & 1060 & 2.8 \\
\hline & 70 & 357 & 229 & 35 & 66 & 2.5 & 638 & 2.7 \\
\hline & 77 & 417 & 267 & 38 & 70 & 45 & 619 & 2.6 \\
\hline
\end{tabular}

Table 13. Trace metal concentrations ( $\mu \mathrm{g} \mathrm{g}^{-1}$ dry wt, Fe as $\%$ dry $w t$ ) in the top $1 \mathrm{~cm}$ of sediments, from 4 boxes in each of the 4 mesocosm basins. Samples taken after 3 mo of control, low, medium and high levels of water dosing of a coppercontaining contaminant

\begin{tabular}{|c|c|c|c|c|c|c|}
\hline Basin & $\mathrm{Cu}$ & $\mathrm{Zn}$ & $\mathrm{Fe}$ & $\mathrm{M} n$ & $\mathrm{Cd}$ & $\mathrm{Pb}$ \\
\hline \multirow[t]{4}{*}{ c } & 32.6 & 142 & 1.38 & 370 & 14 & $6 i i$ \\
\hline & 29.2 & 131 & 1.38 & 363 & 14 & ito \\
\hline & 22.5 & 113 & 1.39 & 453 & 10 & 42 \\
\hline & 45.4 & 142 & 1.23 & 530 & 1.2 & 71 \\
\hline \multirow[t]{4}{*}{ L } & 36.7 & 163 & 1.52 & 326 & 1.5 & 70 \\
\hline & 38.6 & 148 & 1.31 & 407 & 1.6 & 77 \\
\hline & 25.4 & 123 & 1.36 & 440 & 1.0 & 48 \\
\hline & 48.0 & 164 & 1.44 & 431 & 1.4 & 82 \\
\hline \multirow[t]{4}{*}{$M$} & 36.9 & 141 & 1.39 & 538 & 1.3 & 64 \\
\hline & 27.8 & 121 & 1.47 & 380 & 1.4 & 51 \\
\hline & 42.2 & 146 & 1.36 & 366 & 07 & 73 \\
\hline & 45.8 & 146 & 141 & 436 & 14 & 72 \\
\hline \multirow[t]{4}{*}{$\mathrm{H}$} & 433 & 135 & 1.53 & 490 & 1.2 & 64 \\
\hline & 27.0 & 96 & 1.32 & 447 & 1.6 & 42 \\
\hline & 45.5 & 145 & 1.36 & 327 & 1.3 & 72 \\
\hline & 41.8 & $: 29$ & 1.26 & 517 & 12 & 64 \\
\hline
\end{tabular}


Table 14. Platichthys flesus. Metal content $\left(\mu \mathrm{g} \mathrm{g}^{-1}\right.$ dry wt) of liver and kidney samples from individual flounder, collected at Sites 1 to 4 on 11 to 14 Aug (liver samples only, for Site 4). Numbers in parentheses: detection limits

\begin{tabular}{|c|c|c|c|c|c|c|c|}
\hline Site & & $\mathrm{Cu}$ & $\mathrm{Zn}$ & $\mathrm{Fe}$ & $\mathrm{Mn}$ & $\mathrm{Cd}$ & $\mathrm{Pb}$ \\
\hline \multirow[t]{12}{*}{1} & Liver & 36.6 & 99 & 208 & 1.51 & 0.28 & (0.4) \\
\hline & & 13.3 & 87 & 152 & 1.69 & 0.55 & 0.8 \\
\hline & & 30.5 & 86 & 178 & 2.12 & 0.35 & 1.2 \\
\hline & & 42.3 & 110 & 370 & 2.84 & 0.42 & 0.6 \\
\hline & & 29.0 & 191 & 356 & 3.69 & 0.85 & 1.1 \\
\hline & & 39.2 & 124 & 219 & 2.32 & 0.51 & $(0.3 i$ \\
\hline & Kidney & 9.0 & 272 & 706 & 4.02 & 0.66 & 2.2 \\
\hline & & 8.8 & 394 & 600 & 3,56 & 0.65 & 2.2 \\
\hline & & 6.8 & 217 & 536 & 2.02 & 0.55 & 2.8 \\
\hline & & 7.3 & 222 & 905 & 2.25 & 0.41 & 2.1 \\
\hline & & 8.1 & 189 & 751 & 333 & 0.46 & 2.3 \\
\hline & & 8.6 & 145 & 654 & 2.30 & 0.63 & (1.1) \\
\hline \multirow[t]{15}{*}{2} & Liver & 45.3 & 112 & 340 & 2.22 & 0.38 & 1.8 \\
\hline & & 61.9 & 160 & 310 & $2.4 \mathrm{t}$ & 0.33 & 1.1 \\
\hline & & 29.3 & 124 & 316 & 173 & 0.32 & 1.1 \\
\hline & & 40.7 & 113 & 297 & 198 & 0.31 & 1.6 \\
\hline & & 69.5 & 159 & 417 & 5.68 & 2.36 & 7.8 \\
\hline & & 56.8 & 180 & 722 & 6.97 & 1.45 & 4.8 \\
\hline & & 46.1 & 153 & 472 & 4.25 & 2.35 & 11.7 \\
\hline & Kidney & 11.7 & 200 & 396 & 4.21 & 0.87 & 2.9 \\
\hline & & 10.0 & 201 & 914 & 3.22 & 0.49 & 2.0 \\
\hline & & 8.1 & 254 & 577 & 3.37 & 0.70 & 2.3 \\
\hline & & 9.1 & 199 & 1009 & 3.83 & 0.49 & 4.0 \\
\hline & & 7.0 & 196 & 767 & 3.87 & 0.61 & 3.0 \\
\hline & & 5.8 & 199 & 405 & 5.84 & 144 & $(3.6)$ \\
\hline & & 9.5 & 236 & 395 & 4.85 & 101 & 7.5 \\
\hline & & 7.3 & 490 & 576 & 4.92 & 136 & 10.1 \\
\hline \multirow[t]{16}{*}{3} & Liver & 31.6 & 61 & 70 & 1.82 & $(0.12)$ & 14 \\
\hline & & 49.1 & 76 & 151 & 2.08 & 0.21 & 12 \\
\hline & & 16.7 & 58 & 279 & 2.14 & 0.39 & 1.5 \\
\hline & & 28.1 & 68 & 170 & 2.40 & 0.34 & 0.8 \\
\hline & & 56.8 & 90 & 198 & 1.96 & 0.35 & 1.4 \\
\hline & & 28.1 & 84 & 182 & 1.39 & 025 & 1.4 \\
\hline & & 36.6 & 104 & 97 & 2.84 & 043 & 1.4 \\
\hline & & 27.2 & 78 & 83 & 1.83 & 0.22 & 1.1 \\
\hline & Kidrey & 5.4 & 160 & 434 & 4.25 & 098 & 3.7 \\
\hline & & 9.5 & 384 & 495 & 7.16 & 077 & 6.5 \\
\hline & & 8.0 & 165 & 558 & 3.63 & 0.65 & 3.6 \\
\hline & & 6.3 & 264 & 700 & 4.23 & 065 & 7.3 \\
\hline & & 7.9 & 345 & 450 & 3.77 & 0.61 & 4.7 \\
\hline & & 7.3 & 75 & 373 & 5.18 & 0.50 & 3.8 \\
\hline & & 6.0 & 184 & 492 & 4.43 & $0,6.5$ & 6.2 \\
\hline & & 9.8 & 237 & 343 & 5.24 & 0.53 & 81 \\
\hline \multirow[t]{4}{*}{4} & Liver & 24.2 & 192 & 207 & 2.08 & 0.67 & 47 \\
\hline & & 42.3 & 241 & 98 & 1.92 & 0.69 & 5.4 \\
\hline & & 48.8 & 261 & 275 & 2.68 & 0.26 & 55 \\
\hline & & 33.2 & 249 & 311 & 2.77 & 103 & 4.4 \\
\hline
\end{tabular}

Table 15. Platichthys flesus. Metal content $\left(\mu \mathrm{g} \mathrm{g}^{-1} \mathrm{dry}\right.$ wt) of liver samples from individual flounder in mesocosm basins: fish were sampled on 18 to 21 Aug 1986 after an exposure period of about 16 wk. Hyphen: missing data

\begin{tabular}{|c|c|c|c|c|c|c|}
\hline Basin & $\mathrm{Cu}$ & $2 n$ & $\mathrm{Fe}$ & $\mathrm{Mn}$ & $\mathrm{Cd}$ & $\mathrm{Pb}$ \\
\hline \multirow[t]{4}{*}{ C } & 29.1 & 87 & 698 & 2.72 & 0.91 & 5.8 \\
\hline & 1.8 .7 & 68 & 102 & 231 & 0.82 & 5.1 \\
\hline & 230 & 317 & 481 & 303 & 0.83 & 56 \\
\hline & 41.3 & 139 & - & 7.62 & 1.79 & 14.1 \\
\hline \multirow[t]{4}{*}{$\mathrm{L}$} & 23.4 & 59 & 363 & 2.95 & 0.54 & 5.7 \\
\hline & 19.4 & 73 & 299 & 1.98 & 078 & 49 \\
\hline & 360 & 92 & 300 & 256 & 0.80 & 6.3 \\
\hline & 31.9 & $=$ & 279 & 4.05 & 0.89 & 16.3 \\
\hline \multirow[t]{4}{*}{$M$} & 79.9 & 338 & 1790 & 2.68 & 2.89 & 7.6 \\
\hline & 25.2 & 158 & 147 & 2.75 & 0.62 & 4.6 \\
\hline & 102.4 & 487 & 1163 & 22.83 & 2.74 & 14.5 \\
\hline & 15.4 & 65 & 205 & 2.21 & 0.66 & 6.3 \\
\hline \multirow[t]{4}{*}{$\mathrm{H}$} & 19.2 & 103 & 625 & 4.81 & 1.20 & 8.8 \\
\hline & 276 & 293 & 309 & 1.94 & 0.80 & 4.9 \\
\hline & 20.3 & 80 & 195 & 2.88 & 0.63 & 5.8 \\
\hline & 16.2 & 63 & 184 & 2.60 & 071 & 53 \\
\hline
\end{tabular}

Table 16. Mytilus edulis. Mussel whole tissue metal concentrations ( $\mathrm{g} \mathrm{g} \mathrm{g}^{-1}$ dry wt) from Field sites 1 to 4, collected 11 to 14 Aug 1986 (2 replicate pools of 10 ind. each)

\begin{tabular}{|ccccccc|}
\hline \multirow{2}{*}{ Site } & $\mathrm{Cu}$ & $\mathrm{Zn}$ & $\mathrm{Fe}$ & $\mathrm{Mn}$ & $\mathrm{Cd}$ & $\mathrm{Pb}$ \\
\hline \multirow{2}{*}{1} & 13.9 & 176 & 258 & 25.1 & 1.70 & 2.8 \\
& 14.6 & 210 & 256 & 25.9 & 1.39 & 4.1 \\
\multirow{2}{*}{2} & 15.3 & 144 & 293 & 61.1 & 2.32 & 6.3 \\
& 179 & 154 & 324 & 59.1 & 2.61 & 6.9 \\
\multirow{2}{*}{3} & 15.9 & 127 & 580 & 42.7 & 2.94 & 6.6 \\
& 170 & 170 & 583 & 33.3 & 2.49 & 6.4 \\
& 16.4 & 127 & 417 & 70.0 & 2.51 & 7.1 \\
& 17.6 & 173 & 379 & 73.9 & 2.70 & 8.7 \\
& & & & & &
\end{tabular}

Table 17. Mytilus edulis. Metal concentrations ( $\mu \mathrm{g} \mathrm{g}^{-1}$ dry wt) of mussel whole tissues and digestive glands from the mesocosm experiment. Also given are dates on which mussel stocks were placed in the contaminant-dosed basins, and on which sampling took place. Values are of single replicates, each a pool of 10 individuals (except for digestive gland samples from $C, L$ and $M$, for which pool size was 30 )

\begin{tabular}{|c|c|c|c|c|c|c|c|c|}
\hline Basin & $\begin{array}{c}\text { Stock date } \\
(1986)\end{array}$ & $\begin{array}{c}\text { Sample date } \\
\text { (1986) }\end{array}$ & $\mathrm{Cu}$ & $\mathrm{Zn}$ & $\mathrm{Fe}$ & $\mathrm{Mn}$ & $\mathrm{Cd}$ & $\mathrm{Pb}$ \\
\hline \multicolumn{9}{|c|}{ Whole } \\
\hline$c$ & $25 \mathrm{Apr}$ & 13 Jun & 7.8 & 114 & 190 & 13.7 & 1.1 & 2.7 \\
\hline L & $25 \mathrm{Apt}$ & 13 Jun & 10.3 & 147 & 148 & 107 & 0.8 & 2.8 \\
\hline$M$ & 25 Apr & 13 Jun & 17.8 & 156 & 121 & 10.6 & 1.1 & 2.9 \\
\hline $\mathrm{H}$ & $25 \mathrm{Apr}$ & 13 Jun & 53.1 & 86 & 67 & 5.6 & 0.9 & 2.2 \\
\hline $\mathrm{H}$ & $25 \mathrm{Apt}$ & $13 \mathrm{Jun}$ & 47.0 & 78 & 83 & 7.4 & 0.8 & 2.5 \\
\hline$C$ & $25 \mathrm{Apr}$ & 4 Aug & 7.3 & 158 & 217 & 9.8 & 1.2 & 4.9 \\
\hline L & $25 \mathrm{Apr}$ & 4 Aug & 16.3 & 159 & 389 & 174 & 1.3 & 4.0 \\
\hline$M$ & 25 Арг & 4 Aug & 26.8 & 155 & 194 & 9.6 & 1.2 & 3.9 \\
\hline$c$ & $18 \mathrm{Jul}$ & 4 Aug & 6.6 & 58 & 173 & 7.2 & 11 & 3.8 \\
\hline L & $18 \mathrm{Jul}$ & 4 Aug & 9.9 & 102 & 243 & 9.3 & 11 & 3.3 \\
\hline$M$ & $18 \mathrm{Jul}$ & 4 Aug & 14.1 & 88 & 218 & 7.8 & 1.1 & 4.0 \\
\hline $\mathrm{H}$ & $18 \mathrm{Jul}$ & 4 Aug & 59.0 & 91 & 155 & 4.7 & 1.2 & 31 \\
\hline \multicolumn{9}{|c|}{ D Gland } \\
\hline C & $25 \mathrm{Apr}$ & $19 \mathrm{Aug}$ & 14.8 & 175 & 458 & 23.8 & 1.3 & 50 \\
\hline L & $25 \mathrm{Apr}$ & $20 \mathrm{Aug}$ & 27.3 & 196 & 667 & 21.3 & 1.8 & 33 \\
\hline$M$ & $25 \mathrm{Apr}$ & $18 \mathrm{Aug}$ & 35.9 & 171 & 365 & 253 & 1.3 & 31 \\
\hline $\mathrm{H}$ & $18 \mathrm{Jul}$ & 11 Aug & 79.5 & 100 & 128 & 4.3 & 1.5 & 3.4 \\
\hline
\end{tabular}

Table 18. Carcinus maenas. Whole tissue metal concentrations ( $\mathrm{g} \mathrm{g} \mathrm{g}^{-1}$ dry $w \mathrm{t}$ ) from mesocosm basins. Crabs dosed from 12 May 1986 and samples taken on 4 Aug 1986 (2 replicate pools of 4 ind. each

\begin{tabular}{|crrrrrr}
\hline Basin. & $\mathrm{Cu}$ & $\mathrm{Zn}$ & $\mathrm{Fe}$ & $\mathrm{Mn}$ & $\mathrm{Cd}$ & $\mathrm{Pb}$ \\
\hline $\mathrm{C}$ & 96 & 173 & 1773 & 2036 & 1.49 & 6.8 \\
& 97 & 165 & 416 & 14.61 & 1.58 & 8.8 \\
$\mathrm{~L}$ & 70 & 174 & 422 & 14.81 & 1.23 & 7.0 \\
& 88 & 189 & 335 & 9.76 & 1.04 & 6.7 \\
$\mathrm{M}$ & 131 & 231 & 569 & 14.70 & 1.46 & 9.0 \\
& 144 & 176 & 556 & 8.30 & 1.17 & 7.4 \\
$\mathrm{H}$ & 127 & 179 & 240 & 7.42 & 1.49 & 7.8 \\
& 113 & 172 & 307 & 9.20 & 1.04 & 6.1 \\
& & & & & & \\
\hline
\end{tabular}




\section{CHEMISTRY: AROMATIC HYDROCARBONS}

\section{c}

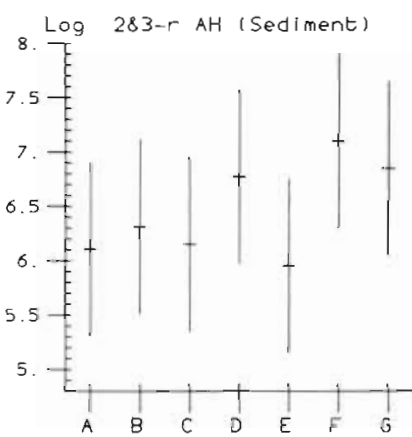

e

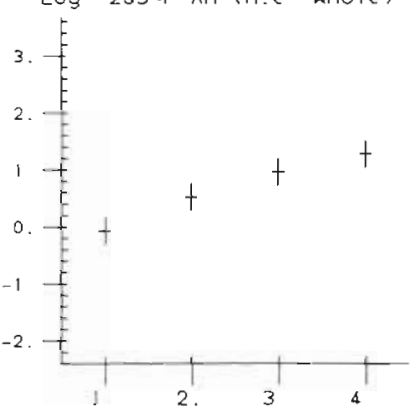

\section{d}

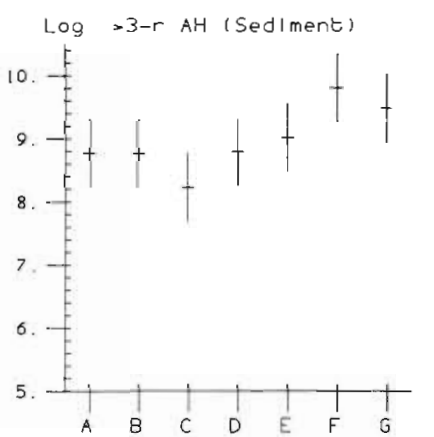

$f$

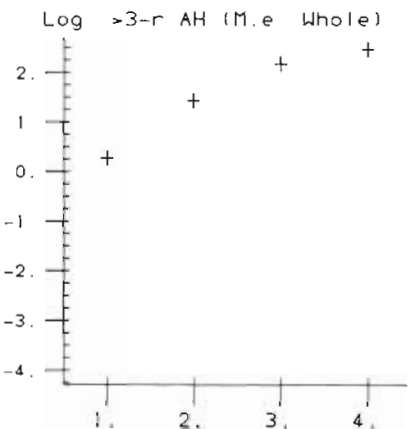

a

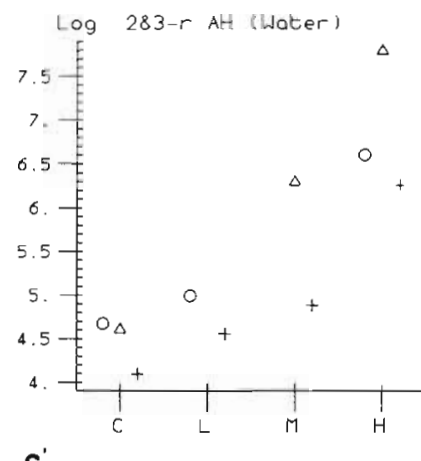

$\mathbf{c}^{\prime}$

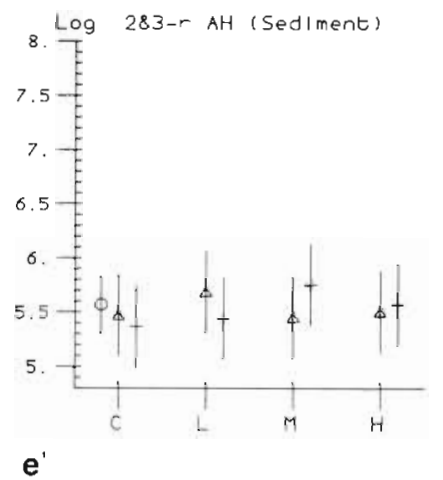

e

Log 283-r AH (M.e. Whole) Log $>3-r$ AH (M.e Whole)

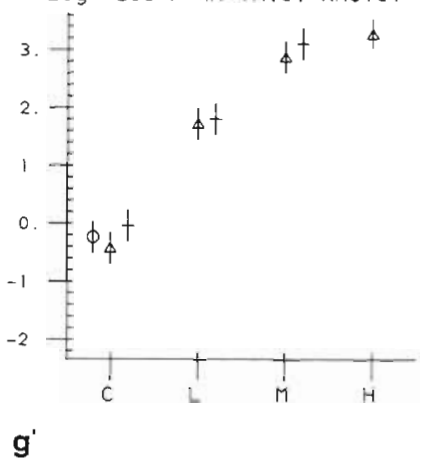

$\mathbf{g}^{\prime}$

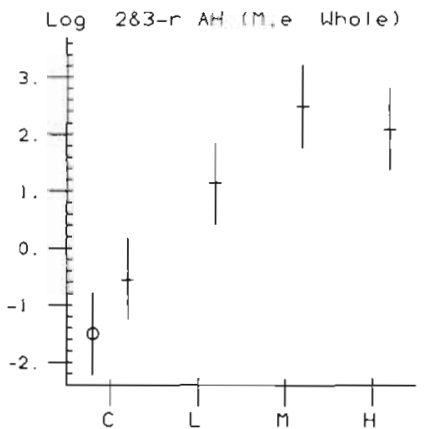

$b^{\prime}$

Log $>3-r$ AH (Water)

$3.5+0$

3.

2.5 -

2.

$1.5 \frac{1}{5}$

0.5

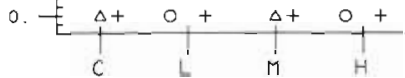

$\mathbf{d}^{\prime}$

Log $>3-r$ AH (Sediment

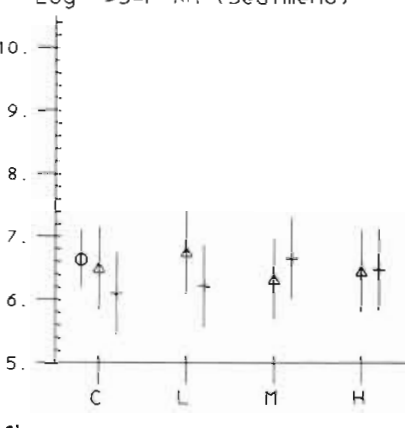

$f^{\prime}$

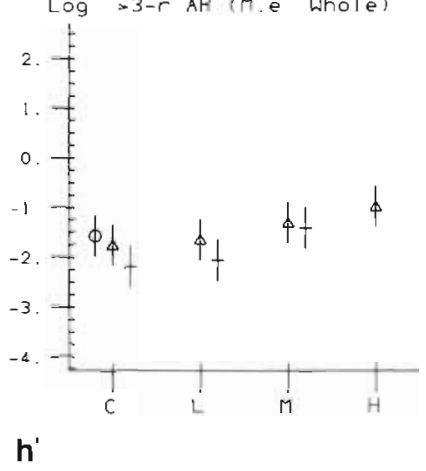

Log $>3-r$ AH (M.e. Whole)

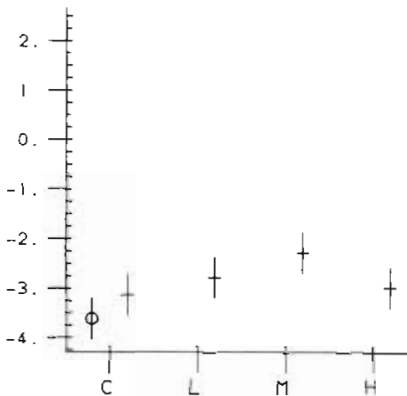

Fig. 1. Aromatic hydrocarbons. (a'), (b') Basin water, sampled at 22 May (o), $19 \mathrm{Jun}(\Delta), 12 \mathrm{Jul}(+)$; (c), (d) sediment, for mesocosm, sampled at 16 May $(0), 13$ Jun ( $\Delta), 7 \mathrm{Jul}(+)$; (e), (f) Mytilus edulis, for mesocosm, individuals stocked in basins on 25 Apr and sampled at 16 May (o), $13 \mathrm{Jun}(\Delta), 4$ Aug $(+) ;\left(g^{\prime}\right),\left(h^{\prime}\right)$ mussels stocked $18 \mathrm{Jul}$ and sampled from stock field population at $7 \mathrm{Jul}(0)$ and basins at $4 \mathrm{Aug}(+)$. Units: (a), (b) $\mathrm{ng} \mathrm{l}^{-1}$; (c), (d) $\mathrm{ng} \mathrm{g}^{-1}$ dry wt; (e) to (h) $\mu \mathrm{g} \mathrm{g}^{-1}$ dry wt. Source: (a) to (h) Klungsøyr et al. (1988) except $\left(c^{\prime}\right),\left(d^{\prime}\right)$ NIVA. 
FIELD

\section{CHEMISTRY: AROMATIC HYDROCARBONS}
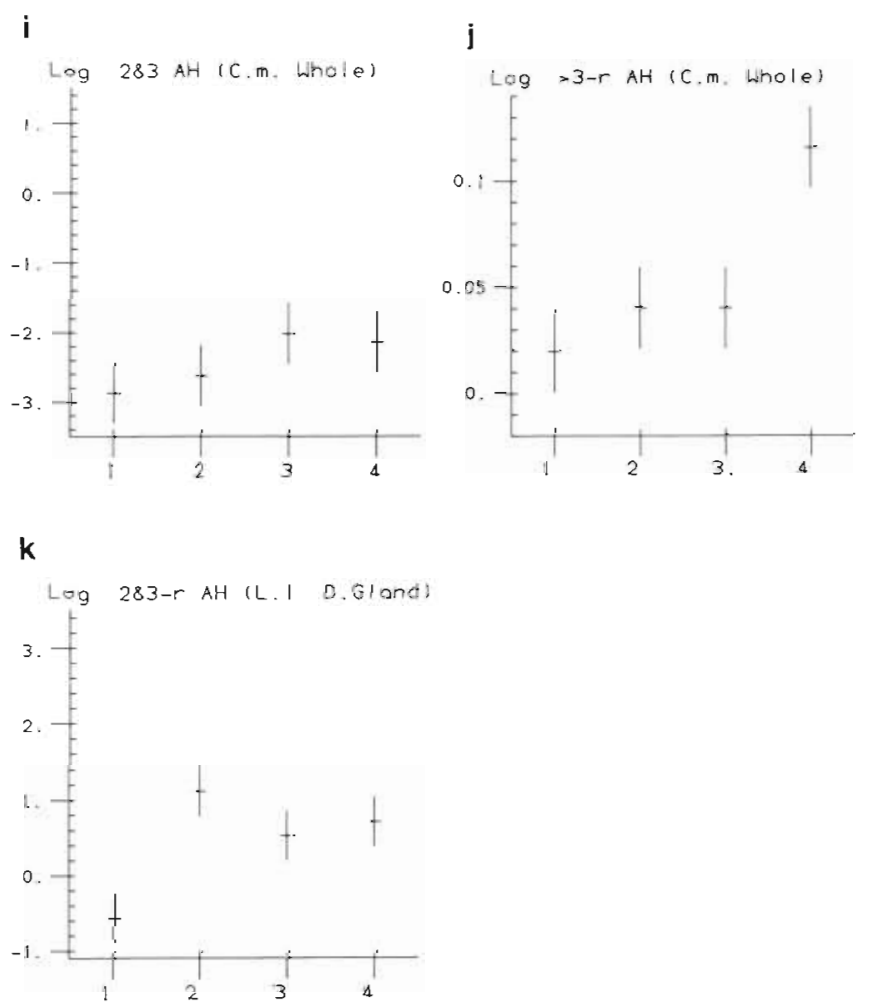

MESOCOSM
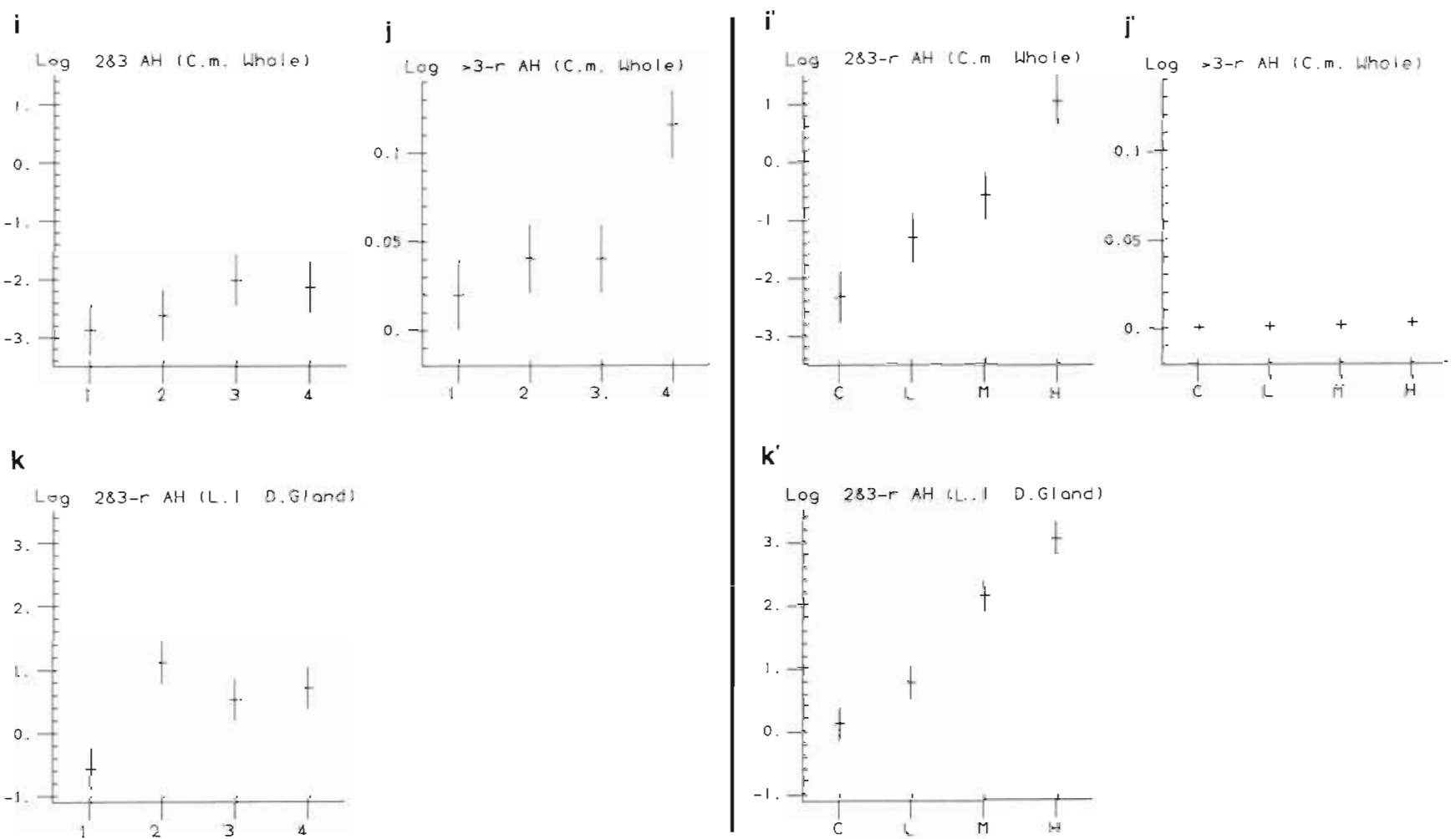

Fig. 1 (continued). Aromatic hydrocarbons. (i), (j) Carcinus maenas, sampled August; (k) Littorina littorea, sampled August ('total' 2- \& 3-ring AH measurements not comparable with GC/MS analysis of other material). Units: (i), (j) $\mu \mathrm{g} \mathrm{g} \mathrm{g}^{-1} \mathrm{dry}_{\mathrm{wt}}(\mathrm{k}) \mu \mathrm{g} \mathrm{g} \mathrm{g}^{-1}$ wet wt. Source: (i), (j) Klungsøyr et al. (1988); (k) Livingstone (1988)

\section{CHEMISTRY: CHLORINATED HYDROCARBONS}

a

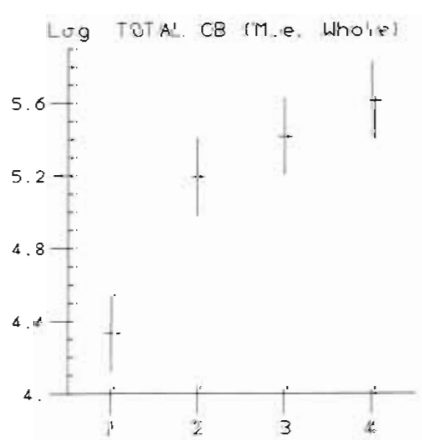

d

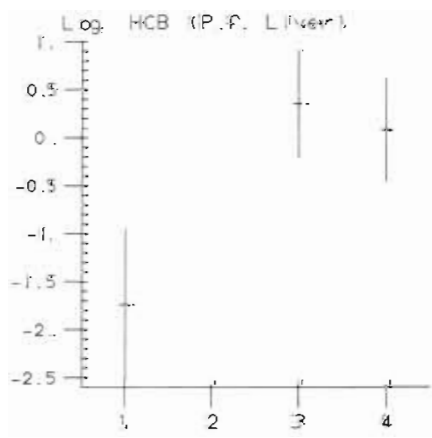

b

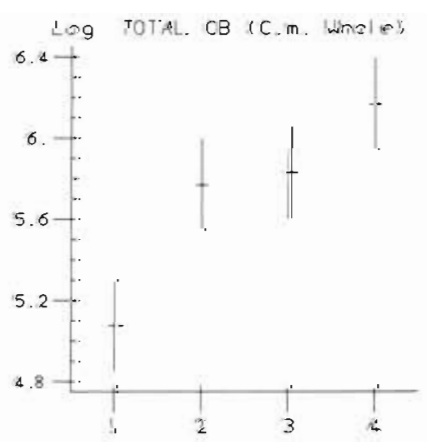

e

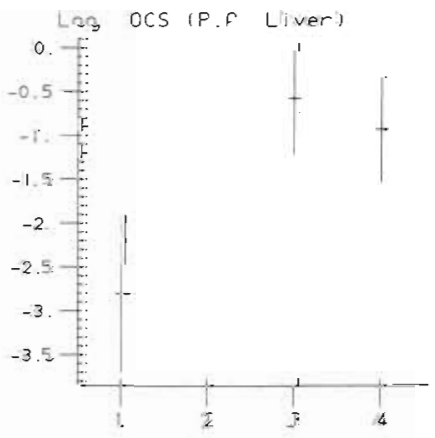

\section{c}

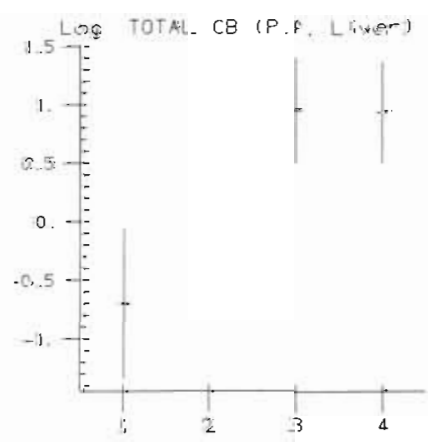

FIELD

Fig. 2. Chlorinated hydrocarbons. (a), (b) PCBs for Mytilus edulis and Carcinus maenas (estimated total of all congeners): (c) PCBs for Platichthys flesus (totals of a subset of 28 congeners); (e), (f) hexachlorobenzene and octachlorostyrene for $P$. flesus. Units: (a), (b) ng $\mathrm{g}^{-1}$ dry wt; (c) to (e) $\mu \mathrm{g} \mathrm{g}^{-1}$ wet wt. Source: (a), (b) Klungsøyr et al. (1988); (c) to (e) Addison \& Edwards (1988) 


\section{CHEMISTRY: METALS}

FIELD

MESOCOSM

a

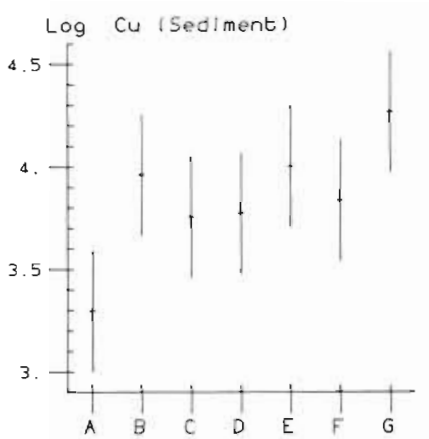

d

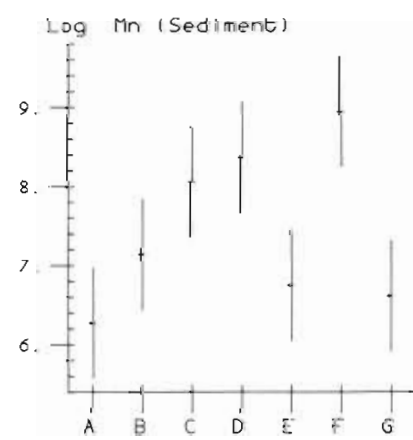

g

Log Cu (P.F Liverskioney)

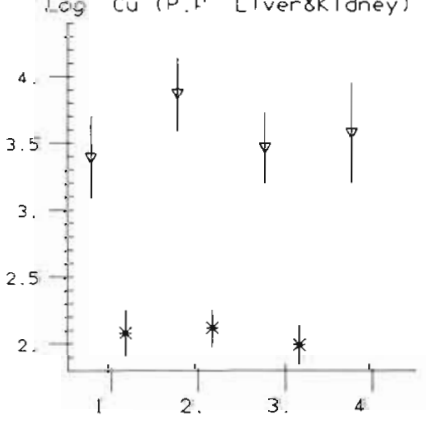

j

Log Mn (P.f Liver\&Kidney)

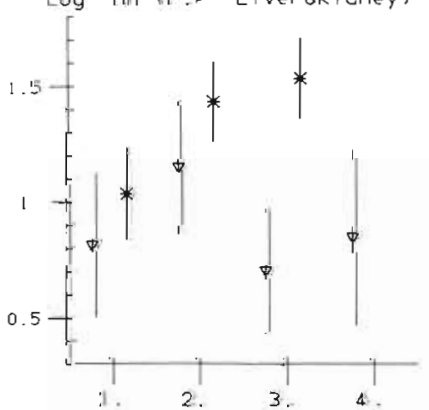

b

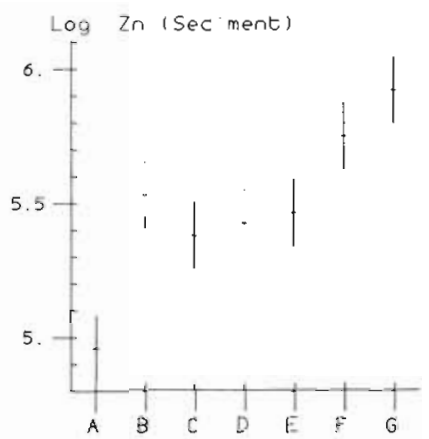

e

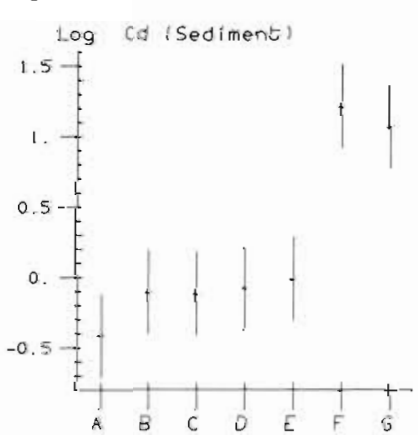

$\mathrm{h}$

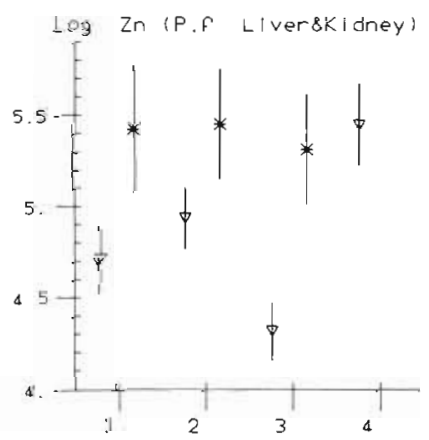

k

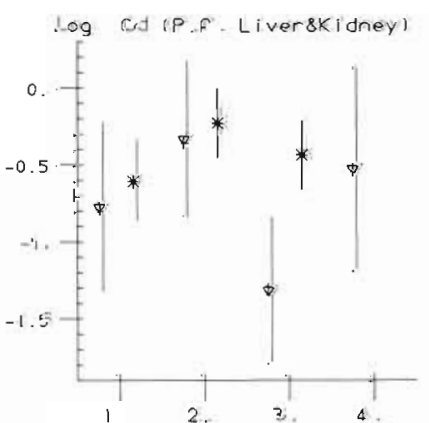

c

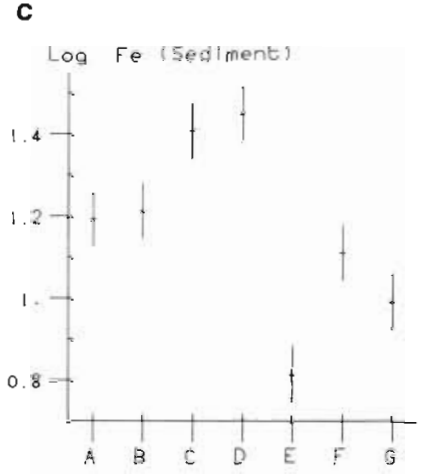

t

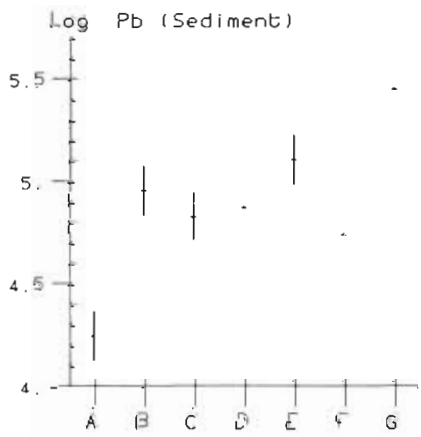

i

Log Fe (P.,. Liverskidney)

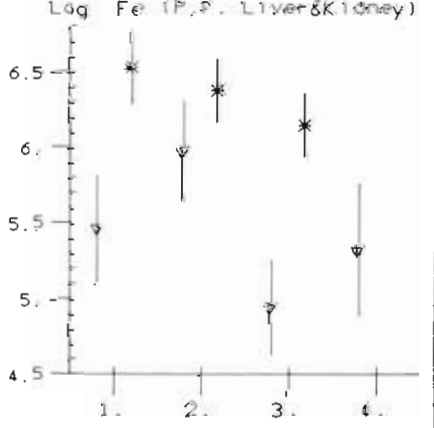

I

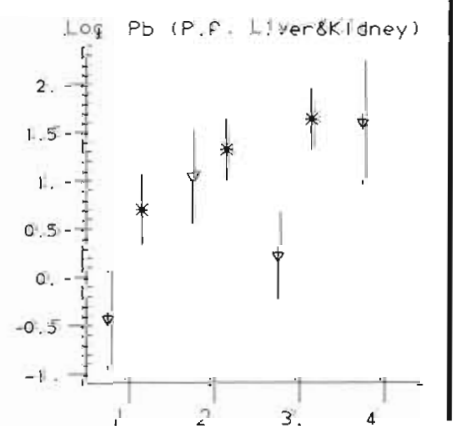

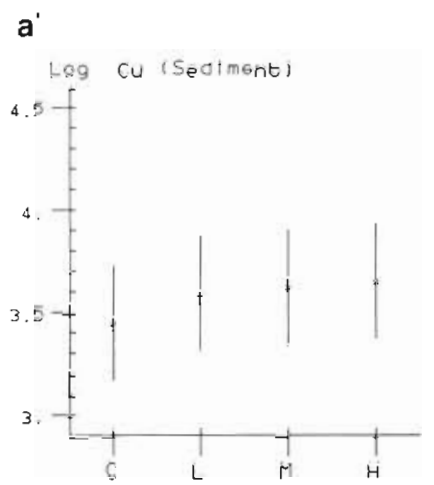

$\mathbf{g}^{\prime}$

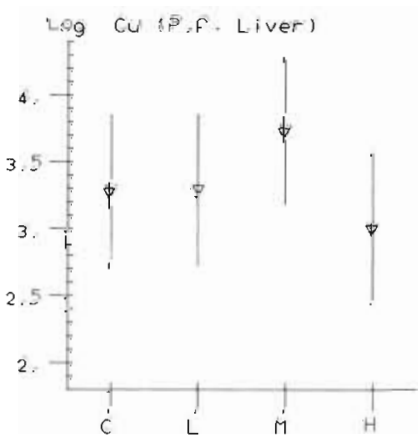

Fig. 3. Trace metals. (a) to (f) Sediments, field samples at $22 \mathrm{Jan}$, mesocosm at $10 \mathrm{Jul}$ ( $\mathrm{g}$ ) to (l) Platichthys flesus liver ( $\nabla$ ) and kidney (*), sampled August. Units: (a) to (l) $\mu \mathrm{g} \mathrm{g}^{-1}$ dry wt. Source: Abdullah \& Steffenak (1988) 


\section{CHEMISTRY: METALS}

FIELD

$\mathrm{m}$

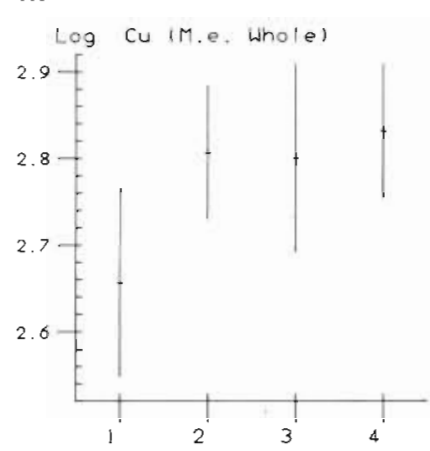

p

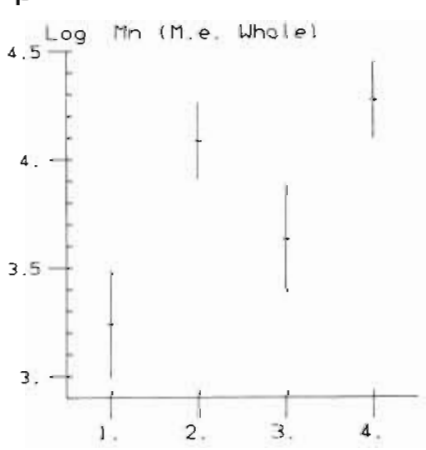

s

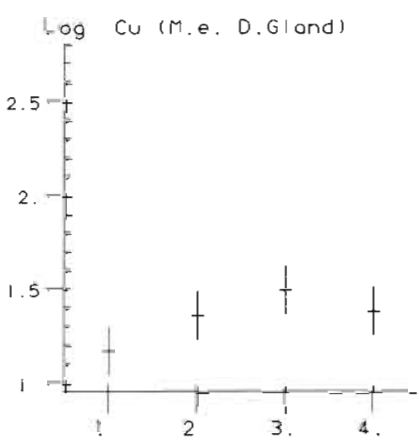

n

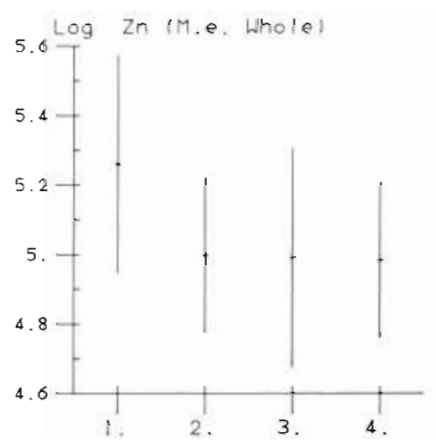

q

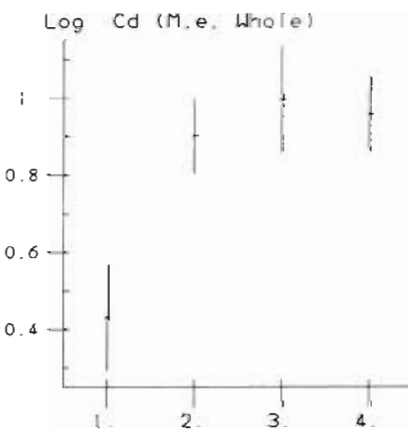

t

3.8 Log Zn (M.e. D.Gland)

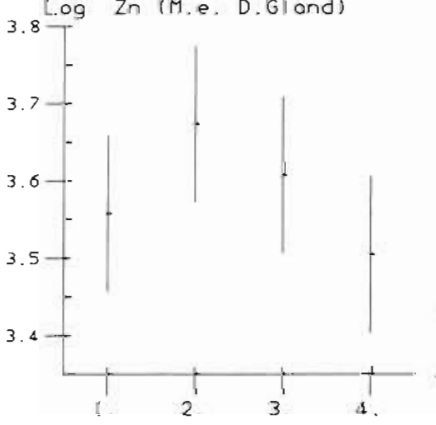

o

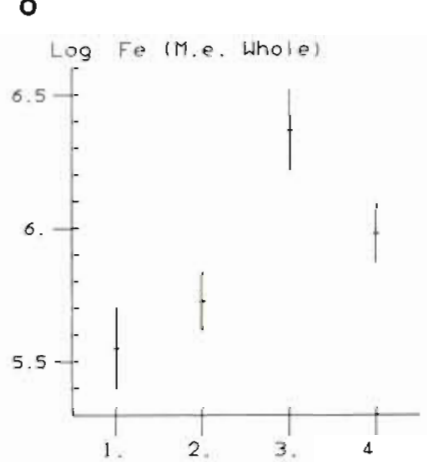

$\mathbf{r}$

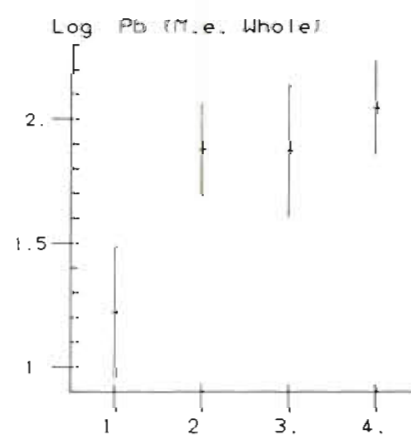

$\mathbf{u}$

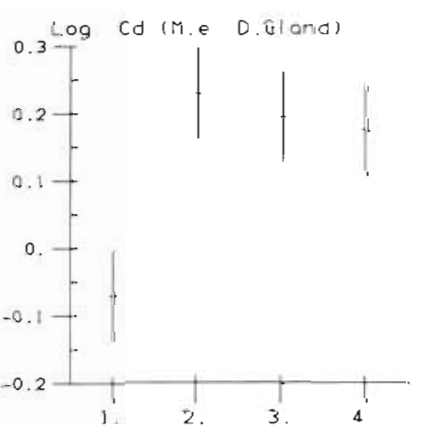

$\mathrm{m}^{\prime}$

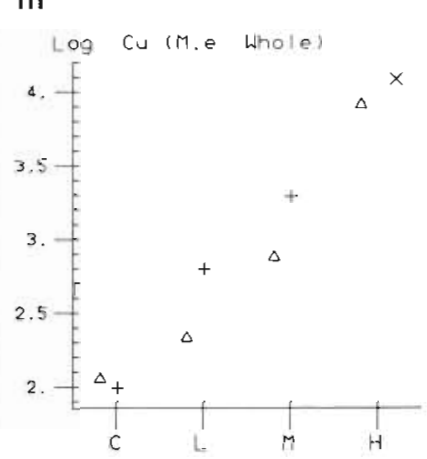

$s^{\prime}$
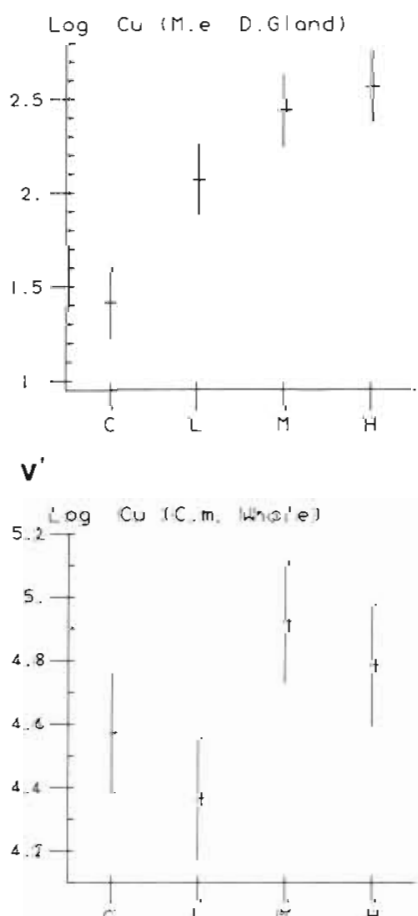

Fig. 3 (continued). Trace metals. (m) to (r) Mytilus edulis (whole tissues), sampled August; (m') mussels stocked in basin on 25 Apr, sampled at $13 \mathrm{Jun}(\measuredangle)$ and $4 \mathrm{Aug}(+)$, except for H basin sampled at 4 Aug $(x)$ which were mussels restocked at $18 \mathrm{Jul} ;$ (s) to (u) mussels (digestive gland), sampled Aug; (v) Carcinus maenas, sampled Aug. Units: (m) to (r), (v) $\mu \mathrm{g} \mathrm{g}^{-1} \mathrm{dry} \mathrm{wt}$ (s) to (u) $\mu \mathrm{g} \mathrm{g}^{-1} \mathrm{wet}^{\mathrm{m}}$ wt. Source: (m) to (r), (v) Abdullah \& Steffenak (1988); (s) to (u) Viarengo et al. (1988) 